\title{
Output-Adaptive Tetrahedral Cut-Cell Validation for Sonic Boom Prediction
}

\author{
Michael A. Park* \\ NASA Langley Research Center, Hampton, VA 23681 \\ David L. Darmofal ${ }^{\dagger}$ \\ Massachusetts Insitute of Technology, Cambridge, MA 02139
}

\begin{abstract}
A cut-cell approach to Computational Fluid Dynamics (CFD) that utilizes the median dual of a tetrahedral background grid is described. The discrete adjoint is also calculated, which permits adaptation based on improving the calculation of a specified output (off-body pressure signature) in supersonic inviscid flow. These predicted signatures are compared to wind tunnel measurements on and off the configuration centerline 10 body lengths below the model to validate the method for sonic boom prediction. Accurate mid-field sonic boom pressure signatures are calculated with the Euler equations without the use of hybrid grid or signature propagation methods. Highly-refined, shock-aligned anisotropic grids were produced by this method from coarse isotropic grids created without prior knowledge of shock locations. A heuristic reconstruction limiter provided stable flow and adjoint solution schemes while producing similar signatures to Barth-Jespersen and Venkatakrishnan limiters. The use of cut-cells with an output-based adaptive scheme completely automated this accurate prediction capability after a triangular mesh is generated for the cut surface. This automation drastically reduces the manual intervention required by existing methods.
\end{abstract}

\section{Introduction}

The acceptance of an aircraft's sonic boom to the general population is a requirement for supersonic flights over land and therefore the commercial viability of a supersonic transport. Predicting how sonic boom signatures are perceived is a challenging task that requires the prediction of the signature on the ground. This is a task complicated by the long propagation distances, atmosphere variations, and the Earth's turbulent boundary layer. A detailed review of the history and state-of-the-art of sonic boom modeling is provided by Plotkin. ${ }^{1}$

The propagation of a sonic boom is often separated into three logical stages or regions, depicted in Fig. 1, to facilitate analysis. ${ }^{2}$ The nearfield is a region near the aircraft, where shocks are formed and strongly influenced by nonlinear phenomena such as shock-shock interaction, shock curvature, and cross flow. Higher pressure portions of the signature travel faster than lower pressure portions of the signature because of variations in the local speed of sound. This slight speed difference causes the shocks to deform by elongating and coalescing in the mid-field. The signature is also refracted by variations in the atmospheric speed of sound. The signature has become primarily an $\mathrm{N}$-wave in the far-field as a result of

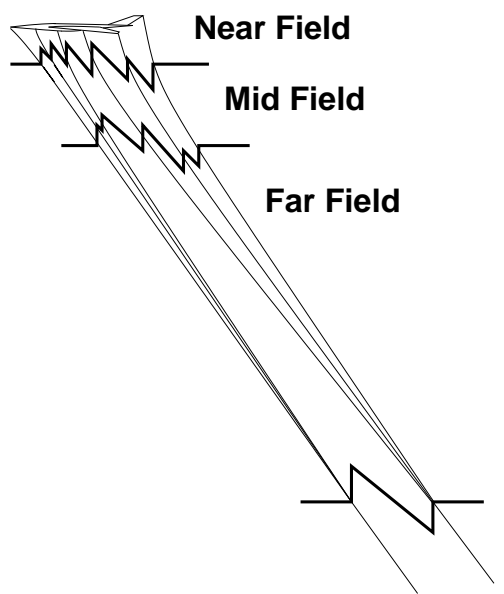

Figure 1. Sonic boom signature propagation zones. this distortion. The boundaries of these regions are case specific.

\footnotetext{
*Research Scientist, Computational AeroSciences Branch, NASA Langley Research Center, MS 128, Hampton, VA 23681, AIAA Member.

${ }^{\dagger}$ Professor, Department of Aeronautic and Astronautics, 77 Massachusetts Ave., 37-401, Cambridge, MA 02139, Senior AIAA Member.
} 
Whitham ${ }^{3,4}$ provides analytic solutions for the signal distortion of slender axisymmetric projectiles. Boom propagation has been implemented in a number of computer programs. ${ }^{5,6}$ Unfortunately, these boom propagation methods are not directly applicable to complex aircraft geometries. Page and Plotkin ${ }^{7}$ applied the multipoles of George ${ }^{8}$ to combine CFD near-field calculation with mid- and far-field boom propagation. This CFD matching multipole propagation technique has been revisited by Rallabhandi and Mavris. ${ }^{9}$

CFD codes have difficulty propagating the relatively weak pressure signatures of a sonic boom to distances beyond the near-field region, where these boom propagation methods are valid. This problem is more acute for unstructured grid methods that are often employed to capture the geometrical complexity of the model, especially if the grids are not aligned with the shocks. To improve alignment, isotropic unstructured grids are stretched to align the tetrahedra with the free stream Mach angle to improve signal propagation for initial grids. ${ }^{10}$ This alignment issue has also given rise to hybrid methods ${ }^{11-13}$ where near-body unstructured grid solutions are interpolated to shock-aligned structured grid methods to increase accuracy. The hybrid methods are hindered by the interpolation process, so adaptive grid methods ${ }^{14,15}$ are employed to improve the accuracy of unstructured grid methods for long propagation distances. These previous adaptive methods have used only primal solution information (Mach and density) to drive the adaptive process.

Adaptive grid methods are designed to specify a resolution request that equidistributes and minimizes error estimates. The improved resolution request is commonly based on local error estimates. ${ }^{16-18}$ Uniformly reducing the errors associated with all local-error sources of the flow may not be optimal from an engineering context, where calculating an output functional (i.e., boom signature) may be of greater concern. An alternative method is to estimate the error in the calculation of a specified engineering output functional. ${ }^{19-22}$ Output error indicators utilize the dual or adjoint solution of an output functional to account for the impact of local error as well as the transport of these local errors throughout the problem domain to improve the calculation of that output functional. This output-adaptive approach has been applied to sonic boom prediction in 2D with discontinuous Galerkin ${ }^{23,24}$ and Cartesian methods. ${ }^{22}$

In this work, anisotropic output-based adaptation to improve an off-body pressure integral is applied to 3D sonic boom prediction. Anisotropically adapted tetrahedral background grids with cut-cells provide extremely robust adaptation mechanics, enabling the automated application of anisotropic output-based adaptation to non-trivial 3D sonic boom problems for the first time. This allows for the entire signature to be calculated or the pressure integral can be restricted to a specific region of interest. The adjoint solution can also provide engineering intuition with a rigorous foundation for design sensitivities ${ }^{25}$ and discretization error estimates. $^{21}$

Cut-cell methods with Cartesian background grids ${ }^{26-29}$ have been very successful for Euler simulations. The regular structure of the Cartesian background grid permits extremely efficient solution schemes. Cartesian background grids have the capability to only provide anisotropic resolution in the Cartesian directions. ${ }^{29}$ Simplex meshes have the ability to stretch the triangular and tetrahedral elements in arbitrary directions. This permits the efficient representation of anisotropic features (i.e., shocks). The cut-cell method is also applicable to simplex meshes. ${ }^{30-32}$ When the constraint of providing a body-fitted grid is removed, the grid adaptation task becomes much simpler. The complexities of adaptation on curved domain boundaries ${ }^{33}$ is eliminated and robustness is dramatically increased.

The 3D anisotropic output-adaptive method for sonic boom prediction has previously been applied to body-fitted grids. ${ }^{34,35}$ This work addresses two of the weaknesses uncovered in the previous work: adjoint iterative convergence for reconstruction limiters and the robustness of body-fitted adaptive mechanics. The current method is validated by comparison to wind tunnel data for representative configurations.

\section{Cut-Cell Determination}

To introduce the 3D cut-cell method a simple 2D example is presented. The control volumes used by the flow solver are the median duals of a triangular mesh. These median duals are constructed by gathering the three dual faces that are inside each primal triangle. Figure 2 shows the three dual faces (dashed lines) for a triangle, which each connect the triangle center to one of the triangle side midpoints. A primal triangular grid is shown in Fig. 3(a). The median duals of this triangular grid are shown in Fig. 3(b). The airfoil geometry is a diamond airfoil, shown with the uncut median dual background grid in Fig. 3(c). The airfoil geometry is Boolean subtracted from this background grid removing the portion of the background grid

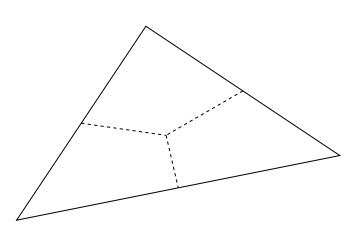

Figure 2. Three dual faces (dashed lines) associated with a single triangle (solid lines). 
that is external to the flow domain, Fig. 3(d).

In the 3D case, the domain of the simulation is constructed by Boolean subtraction of a manifold triangular boundary representation from a background grid. This triangulation can come from many sources. Figure $4(\mathrm{~b})$ is a triangular surface grid of a cylinder constructed on a CAD solid. ${ }^{36}$

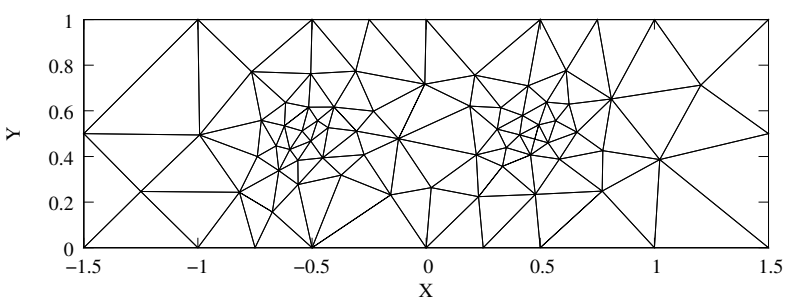

(a) Primal grid.

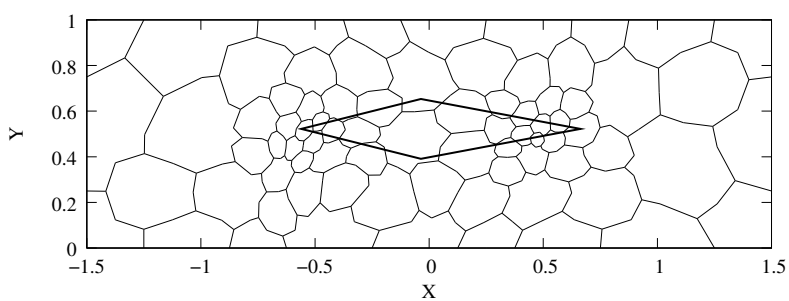

(c) Median dual grid with geometry.

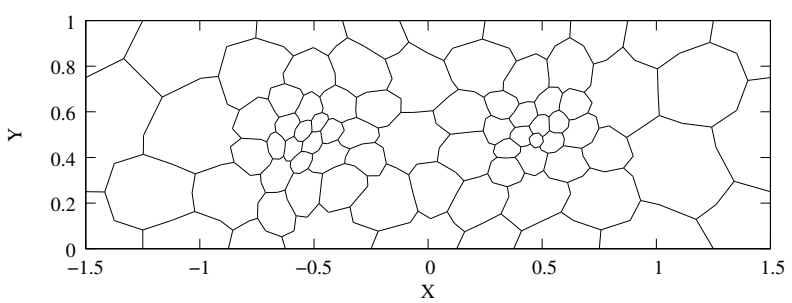

(b) Median dual grid.

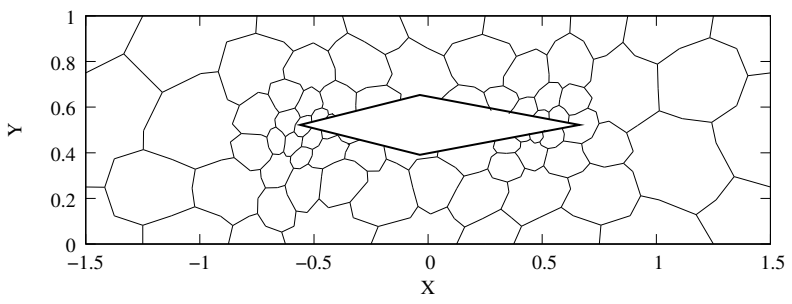

(d) Resulting cut-cell grid.

Figure 3. Cut cell illustration of an diamond airfoil in 2D.

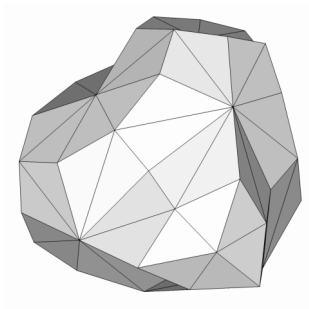

(a) Median dual.

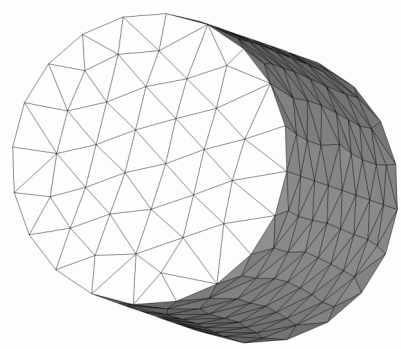

(b) Cylindrical cutting surface.

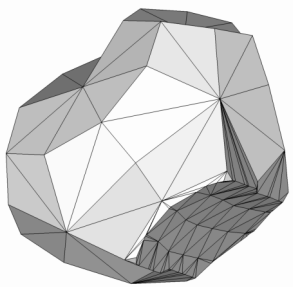

(c) Resulting Polyhedra.

Figure 4. Cylindrical cutting surface and median dual.

The background grid contains closed simplicial polytope control volumes. In 3D, these polyhedra are the median duals of a tetrahedral grid. The 3D median dual about a single primal node is shown in Fig. 4(a). Just as in the 2D case, this dual control volume may not be convex. Each dual polyhedra of a tetrahedral grid contains $\mathcal{O}(100)$ triangles. Figure 5 illustrates the two dual triangles associated with an edge of a primal tetrahedron. There are six edges in a tetrahedron, which contains a total of 12 triangular dual faces shared by the duals at each of its 4 nodes. For robustness and a decrease in execution time and memory usage, a triangular dual face is only represented once in the intersection procedure and shared by the two adjacent control volumes.

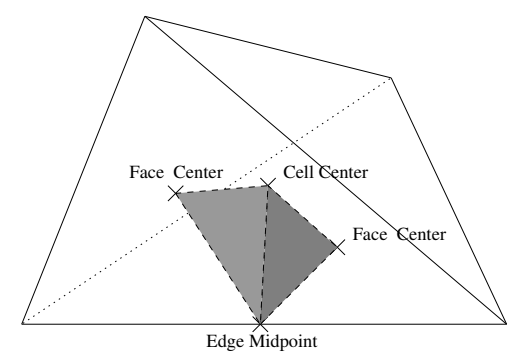

Figure 5. Two dual triangles associated with a single tetrahedral edge. 
Aftosmis, Berger, and Melton ${ }^{28}$ demonstrate a method to perform the Boolean subtraction of two manifold triangular polyhedra (surface grid and each of the background grid control volumes) with a series of triangle-triangle intersections. Their methodology was modified to use only floating point arithmetic by Park. ${ }^{37}$ The result of this subtraction is shown in Fig. 4(c).

\section{Flow and Adjoint Solvers}

Fully Unstructured Navier-Stokes Three-Dimensional (FUN3D) is a suite of codes for finite-volume CFD. ${ }^{38}$ The FUN3D website, http://fun3d.larc.nasa.gov, contains the user manual and an extensive list of references. FUN3D is able to solve incompressible, Euler, and Reynolds-averaged Navier-Stokes (RANS) flow equations, either tightly or loosely coupled to a turbulence model. The Euler equations are used in this study. Domain decomposition is employed to fully exploit the distributed memory of a cluster of computers to increase problem size and reduce the execution time of the simulation process.

The Euler equations are

$$
\begin{aligned}
& \frac{\partial \mathcal{Q}}{\partial t}+\nabla \cdot F=0, \\
& \mathcal{Q}=\left[\begin{array}{c}
\rho \\
\rho u \\
\rho v \\
\rho w \\
E
\end{array}\right], \quad F=\left[\begin{array}{c}
\rho u \\
\rho u^{2}+p \\
\rho u v \\
\rho u w \\
u(p+E)
\end{array}\right] \hat{i}+\left[\begin{array}{c}
\rho v \\
\rho v u \\
\rho v^{2}+p \\
\rho v w \\
v(p+E)
\end{array}\right] \hat{j}+\left[\begin{array}{c}
\rho w \\
\rho w u \\
\rho w v \\
\rho w^{2}+p \\
w(p+E)
\end{array}\right] \hat{k},
\end{aligned}
$$

where $\rho$ is density, $u, v$, and $w$ are velocity, $E$ is total energy per unit volume, and $p$ is pressure. These quantities are related by the ideal gas relation,

$$
p=(\gamma-1)\left(E-\rho \frac{u^{2}+v^{2}+w^{2}}{2}\right),
$$

with the specific heat ratio $\gamma=1.4$ for air.

The divergence theorem is applied over a set of control volumes to produce a finite-volume scheme,

$$
\int_{V_{i}}\left(\frac{\partial \mathcal{Q}}{\partial t}+\nabla \cdot F\right) \mathrm{d} V=V_{i} \frac{\mathrm{d} \mathcal{Q}_{i}}{\mathrm{~d} t}+\int_{\Gamma_{i}} F \cdot \vec{n} \mathrm{~d} \Gamma=0
$$

where $\Gamma_{i}$ are the boundaries of the control volumes with volume $V_{i}$ and $\vec{n}$ is an outward pointing normal. The average of $\mathcal{Q}$ in each control volume is $\mathcal{Q}_{i}$. The flux integration is approximated as,

$$
\int_{\Gamma_{i}} F \cdot \vec{n} \mathrm{~d} \Gamma \approx \sum_{f \in \Gamma_{i}} \mathcal{H}\left(q_{l_{f}}, q_{r_{f}}, \vec{n}_{f}\right) A_{f}=R_{i}(Q),
$$

where $R_{i}$ is the discrete residual for control volume $i$, the summation is over the faces of the control volume. ${ }^{38}$ The van Leer ${ }^{39}$ approximate Riemann solver $\mathcal{H}$ is utilized to compute the flux from the primitive states,

$$
q=\left[\begin{array}{lllll}
\rho & u & v & w & p
\end{array}\right]^{T},
$$

at the borders of the neighboring control volumes, $q_{r f}$ and $q_{l f}$. These face values are reconstructed from cell averages (the reconstruction method is described below). The discrete equations are established simultaneously for each control volume,

$$
V \frac{\mathrm{d} Q}{\mathrm{~d} t}+R(Q)=0
$$

which makes the discrete solution vector $Q \in \mathcal{R}^{5 N}$, discrete residual vector $R \in \mathcal{R}^{5 N}$, and $V=\operatorname{diag}\left(V_{i}\right)$, where $N$ is the number of control volumes. The flux integration scheme (including face state reconstruction from cell averages) is detailed in the following sections.

A backward Euler solution update scheme is employed with a variable pseudo-time step. ${ }^{38}$ An approximate nearest neighbor linearization is utilized to reduce the memory required for the implicit point-iterative method. After the flow solution is known, the discrete adjoint equations ${ }^{25,40}$ are solved to complete the dual problem. The linear adjoint equations are solved with a dual-consistent time-marching method. ${ }^{41,42}$ The dual-consistent solution method guarantees that the adjoint equations will have the same asymptotic convergence rate as the flow equations. 


\section{III.A. Inviscid Flux Integration}

The existing FUN3D body-fitted approach lumps the median dual pieces to a single effective area and normal direction for each edge they surround. ${ }^{43}$ After lumping, all of the inviscid terms are calculated with a loop over edges, which is computationally efficient. Conserved states $Q$, used in the time advancement scheme, are converted to primitive states $q$ for face state reconstruction. The primitive state is extrapolated from the nodes to establish the primitive state at these lumped faces $q_{f}$ using the gradients $\nabla q=\left[q_{x}, q_{y}, q_{z}\right]$, face center $x_{f}$, and node $x_{0}$,

$$
q_{f}=q_{0}+\nabla q\left(x_{f}-x_{0}\right),
$$

for the unlimited scheme. The gradient is reconstructed from the cell averaged state $q_{0}$ with a weighted least squares method, see Park ${ }^{37}$ for details. For the case of supersonic flow, a limiting function is used to reduce the gradient contribution to the reconstruction (see Subsection III.B).

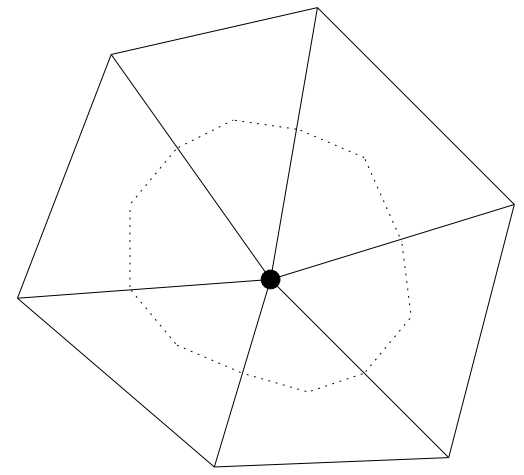

(a) Uncut control volume.

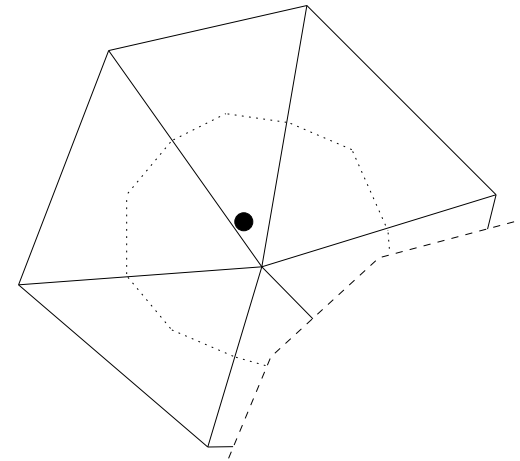

(b) Cut-cell control volume.

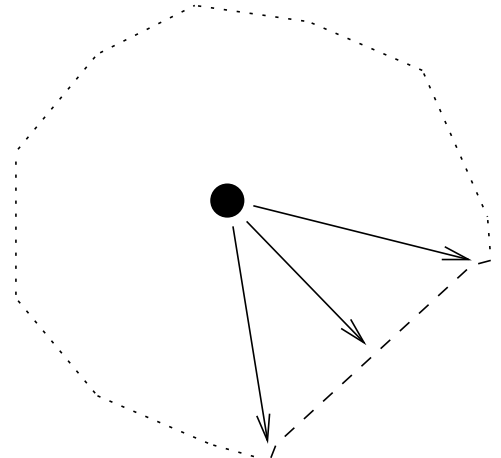

(c) Cut-cell boundary integration.

Figure 6. Dual control volumes, in $2 \mathrm{D}$.

At the completion of cut-cell preprocessing, the dual polyhedra can be classified into three groups: uncut active duals interior to the computational domain, cut duals, and inactive uncut duals exterior to the computational domain. The state is stored at each node in the primal grid, Fig. 6(a) filled circle. All nodes that correspond to dual polyhedra that have been cut or are inactive are removed. A new degree of freedom is inserted at each cut dual polyhedra centroid, Fig. 6(b) filled circle. Multiple degrees of freedom are added when a polyhedra is split into multiple distinct regions by the cut surface.

Once a dual control volume is cut, the approximation that the state is centered at the primal node is removed and the state becomes centered at the control volume centroid. This results in a discontinuous change in location once the control volume is infinitesimally cut. This discontinuous behavior may cause difficulties for shape sensitivities and design. Removing this issue remains a topic for future work, but may be addressed by computing the uncut cell centroids.

The median dual triangles that surround any edges that involve a cut cell are explicitly represented and employed in flux integration. Edges that involve uncut active duals utilize the lumped effective areas and normals of the body-fitted scheme. Cut-cell flux integration requires more work than the body-fitted scheme because there are multiple triangles separating the two control volumes that would be approximated as a single flux evaluation in the body-fitted scheme. It also requires more memory to store the extra triangles that would be approximated as a single effective area. The cut cells are a minority of the control volumes for a typical case, so the additional expense of utilizing cut cells does not dominate the execution time or storage.

The body-fitted, node-based scheme stores the state on the boundary of the domain. The state is interpolated between adjacent boundary nodes to integrate the boundary flux. The boundary flux is described in Section IV. For cut-cell boundary flux integration, the state is extrapolated with the reconstructed gradients from the cell centroids to the boundary face,

$$
q_{b f}=q_{0}+\nabla q\left(x_{b f}-x_{0}\right) .
$$

The boundary of the cut cell from Fig. 6(b) is shown in Fig. 6(c) to illustrate the reconstruction of the 
boundary state $q_{b f}$ for cut-cell boundary flux integration. The $q_{b f}$ is extrapolated to each cut surface piece for integration.

\section{III.B. Reconstruction Limiting}

Barth and Jespersen ${ }^{43}$ introduced limits on an unstructured grid reconstruction scheme to maintain monotonicity. Face reconstruction using a limiter of this form is

$$
q_{f}=q_{0}+\Phi \nabla q\left(x_{f}-x_{0}\right),
$$

where the diagonal matrix limiting function $\Phi$ is computed in each control volume. The same $\Phi$ is employed in all face reconstructions for a given control volume. This type of limiter can compromise the convergence of the flow solution and therefore a dual-consistent adjoint solver. ${ }^{44,45}$ Venkatakrishnan ${ }^{46}$ studied this limiter in its original form as well as with the limiter function held constant (i.e. frozen) after iterative convergence stalls. He proposed a new limiter to improve convergence, but both the frozen scheme and new limiter can result in stalled convergence. The Venkatakrishnan limiter is not monotone, it permits under- and overshoots. Frozen limiters are approximations that impede error estimation, output-based adaptation, adjoint iterative convergence, and design sensitivities. ${ }^{22,34,47}$

In this study, the limiter will be used in the context of an output adaptive scheme that requires the adjoint solution. An exact linearization and steady iterative convergence of the flow and adjoint solvers is paramount to the robustness of the adaptive scheme. This iterative convergence is so critical that the accuracy of the limited scheme will be sacrificed; accuracy will be regained with adaptive grid refinement and alignment. A heuristic edge-based limiter ${ }^{48}$ is utilized to improve the convergence of the flow solver while providing the exact linearization required for adjoint convergence. Concessions are made to improve iterative convergence; it is not total variation diminishing (TVD) or linearity preserving.

The heuristic limiter was developed ${ }^{48}$ by examining its effect on shock capturing for regular and irregular grids and empirically adjusting its formulation to increase the width of shocks. It is a scalar limiting function $\phi$ that considers only the cell-averaged values of pressure and their reconstructed gradients in the cells adjacent to the face being reconstructed. Face reconstruction using a limiter of this form is

$$
q_{f}=q+\phi \nabla q\left(x_{f}-x_{0}\right),
$$

where the scalar limiting function $\phi$ is computed for each face $f$. The same $\phi$ is used for the left $q_{l f}$ and right $q_{r_{f}}$ face reconstructions.

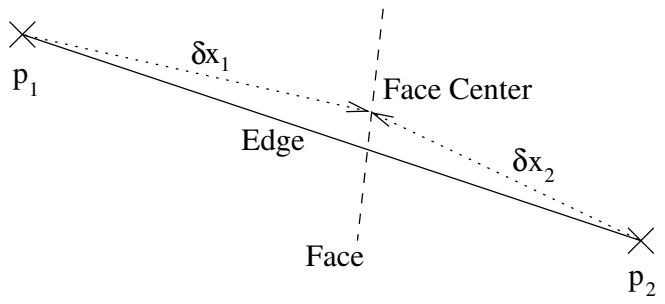

Figure 7. Edge and face geometry.

The basic concept employed in this heuristic limiter is to reduce the reconstruction gradient in locations where the pressure gradients are large relative to pressure. This clearly could result in limiting in regions for which the solution varies linearly (though with large magnitude), however, in combination with adaptation the proposed limiter has been found robust and accurate. The specific form of the limiter relies on a measure of the change in the pressure, $\delta p$. To form $\delta p$, the reconstructed gradient of pressure for the control volumes on the right and left of the face (Fig. 7), $\nabla p_{1}$ and $\nabla p_{2}$, are used with the right and left extrapolation vectors to the face, $\delta x_{1}$ and $\delta x_{2}$,

$$
\delta p=\left\|\begin{array}{c}
\delta x_{1_{x}} \nabla p_{1_{x}}-\delta x_{2_{x}} \nabla p_{2_{x}} \\
\delta x_{1_{y}} \nabla p_{1_{y}}-\delta x_{2_{y}} \nabla p_{2_{y}} \\
\delta x_{1_{z}} \nabla p_{1_{z}}-\delta x_{2_{z}} \nabla p_{2_{z}}
\end{array}\right\| .
$$

This sensor is active for linear functions and does not specifically penalize extrema. The gradient reconstruction is reduced where the the $\delta p$ sensor is large with the intention of spreading the detected jump over 
a number of control volumes. Adaptation will be employed to narrow the width of the discontinuity. The tanh function is employed to smooth the combined nondimensional pressure jump ratio,

$$
\phi_{\text {heuristic }}=1-\tanh \left(\frac{\delta p}{\min \left(p_{1}, p_{2}\right)}\right),
$$

and restricts the limiter to the range $(0,1]$. A tanh function is employed to provide a smoothly varying and differentiable function that enables residual convergence that can be impeded by a non-smooth limiting function. This limiter is active (to some degree) in all regions with pressure variations, so it will not switch on and off intermittently during iterative convergence. The design accuracy of the limited scheme is therefore below second-order. The limiter is more active when the pressure variation is significant as compared to the local pressure.

The cut cells require pressure extrapolated to the boundaries to compute boundary fluxes. This reconstruction requires limiting to prevent unrealizable face states and must be smoothly differentiable to facilitate iterative convergence,

$$
\begin{gathered}
\delta p_{d}=\left\|\begin{array}{c}
\delta x_{x} \nabla p_{x} \\
\delta x_{y} \nabla p_{y} \\
\delta x_{z} \nabla p_{z}
\end{array}\right\|, \\
\phi_{\text {extrapolation }}=1-\tanh \left(\frac{\delta p}{p}\right) .
\end{gathered}
$$

The extrapolation limiter is formulated to mimic the interior face limiter using only the data from the cell adjacent to the boundary.

\section{Boundary Conditions}

The boundary conditions are imposed weakly through the fluxes. The tangential flow boundary condition is implemented with zero velocity normal to the boundary, resulting in the flux,

$$
F_{\text {tangential }}=\left[\begin{array}{c}
0 \\
p n_{x} \\
p n_{y} \\
p n_{z} \\
0
\end{array}\right],
$$

where $p$ is interpolated along or extrapolated to the boundary. The supersonic outflow boundary condition uses the interior state to form the boundary flux. The supersonic inflow boundary condition uses the free stream state $\rho_{\infty}, u_{\infty}, v_{\infty}, w_{\infty}$, and $p_{\infty}$ to form the boundary flux.

The inviscid flow model breaks down at a sharp corner where separation would occur in a physical flow. In the supersonic flow simulations performed in this work, this problem presents itself at blunt trailing edges. To avoid this problem in these regions, a transpiration boundary condition is specified manually. This boundary condition applies free stream velocity state, $u_{\infty}, v_{\infty}$, and $w_{\infty}$ with a density and pressure of $\rho=0.3 \rho_{\infty}$ and $p=0.3 p_{\infty}$. This level of density and pressure is empirically established by examining the solution of a backward facing step with the tangential boundary condition.

\section{Output-Based Adaptation}

Venditti ${ }^{21}$ describes an output-based error estimation and adaptation scheme. To formulate the error estimate, an embedded grid is required. Constructing the entire embedded grid can be infeasible for large $3 \mathrm{D}$ grids and has prevented the use of adjoint error estimation techniques for industrial-sized problems even with a parallel implementation. ${ }^{34}$ While the embedded grid can be formed in sections, this increases the error estimation scheme complexity. Forming a portion or the entire embedded grid is also complicated by the need to respect curved boundaries and recompute the intersection tests of cut cells. These difficulties have motivated the desire to employ only the current grid in the error estimation procedure. A procedure 
is described that obtains an indicator for output adaptation with the current grid, but does not provide a functional error correction.

Park $^{37}$ provides a derivation of the single-grid adaptive indicator by placing it in the context of the embedded grid approach of Venditti. In the interest of brevity, the single-grid error estimation and adaptive indicator is provided without a derivation,

$$
\left[I_{\text {single }}\right]_{\kappa}=\frac{1}{2} \sum_{i=1}^{5}\left\{\left|\left[R^{\lambda}(\hat{\lambda})\right]_{i, \kappa}[\hat{Q}-\bar{Q}]_{i, \kappa}\right|+\left|[\hat{\lambda}-\bar{\lambda}]_{i, \kappa}[R(\hat{Q})]_{i, \kappa}\right|\right\}
$$

It has the same pieces as the Venditti error indicator, where the five conservation equations are contracted by the summation over $i$. The vector $I_{\text {single }} \in \mathcal{R}^{N}$ has a single value for each grid control volume $\kappa$. The $\hat{\lambda}$ and $\hat{Q}$ higher-order reconstructions and the $\bar{\lambda}$, and $\bar{Q}$ lower-order reconstructions on the current grid are described in Park. ${ }^{37}$ The original residual operators are utilized and $\hat{\lambda}$ and $\hat{Q}$ are constructed to make $R^{\lambda}(\hat{\lambda})$ and $R(\hat{Q})$ reliable adaptive indicators. The $\hat{\lambda}$ and $\hat{Q}$ reconstructions are formed with a fit of quadratic functions to cell averaged states and their gradients. The difference between the $\hat{()}$ and $\overline{()}$ reconstructions is intended to provide adequate guidance for the relative distribution of error, not a sharp bound on error.

Venditti ${ }^{21}$ provides a procedure to calculate a new grid spacing request $h$ from the adaptive indicator $I_{\kappa}$ and an error tolerance tol ${ }_{\Omega}$. The adjoint adaptation parameter was also incorporated into an anisotropic Hessian-based framework. This combined approach sets the anisotropy of mesh elements by using the Mach Hessian, and it scales the element size so that the tightest spacing is dictated by the adjoint adaptation parameter. The metric-based grid mechanics is described by Park and Darmofal. ${ }^{37,49}$ It is fully parallelized and interfaces directly with the error estimation process.

\section{Application to Sonic Boom Prediction}

The parallel metric-based adaptation algorithm is applied to sonic boom prediction. More detailed information on these cases, including timing information, is available in Park. ${ }^{37}$ The output for the adaptive procedure of the integral of quadratic pressure deviation over a surface $s$ in the domain,

$$
f=\frac{1}{A_{s}} \iint_{s}\left(\frac{p-p_{\infty}}{p_{\infty}}\right)^{2} \mathrm{~d} s
$$

where $A_{s}$ is the area of the integration surface. This focuses the adaptation on improving the calculation of pressure near this surface. Previous applications have been performed with the integral of pressure deviation. $^{34,35}$ However, the square of this deviation has been shown to produce more accurate signatures with less control volumes. ${ }^{22}$ A cylindrical integration surface, aligned to the $x$-axis, is employed. The extent of the integration surface can be optionally restricted to the interior of a box to focus on a subsection of the cylinder.

The current output-based adaptation approach is validated with wind tunnel measurements. Wind tunnel testing of sonic boom configurations is a challenging task. Wind tunnel models are typically small to obtain pressure signatures a relatively large distance from the model within the finite size of tunnel test sections. Carlson and Morris ${ }^{50}$ present some of difficulties inherent in wind tunnel testing of these small models including extraneous variations in pressure larger than the signals measured. A test apparatus and procedure that mitigates the extraneous spatial and temporal distortions is described. Morgenstern ${ }^{51}$ also documents variations in ambient static pressure wind tunnel measurements that are of the same magnitude as the desired signature measurement.

\section{VI.A. Cone-Cylinder Configuration}

A double cone geometry, denoted "Model 8" in a 1965 wind tunnel report, ${ }^{52}$ is shown in Fig. 8 with a shaded triangular surface grid. This same case was employed to evaluate ${ }^{34}$ and then validate ${ }^{35}$ a parallel adaptive body-fitted grid approach. This configuration has also been used by other researchers to evaluate their signature prediction techniques. ${ }^{12,15}$ The pressure integral output function was defined as a cylinder, six body lengths in radius, centered about the geometry axis. The cylinder is clipped forward of 3 body lengths behind the

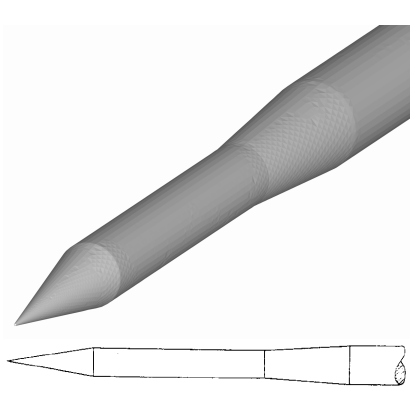

Figure 8. Cone-Cylinder Geometry. 
nose, aft of 9 body lengths behind the nose, and outside of 0.1 body lengths off the centerline to focus only on the region where wind tunnel data is available. The surface grid is Boolean subtracted from a 9-degree wedge-shaped background tetrahedral volume grid. A symmetry plane of the volume grid is shown in Figure 9. The initial grid (4,000 control volumes) was created with no prior knowledge of where the shocks would propagate through the domain, Fig. 9(a). The free stream Mach number is 1.26 and the heuristic limiter is employed during adaptation. The parallel execution scheme used 32 partitions, and the $17^{\text {th }}$ adapted grid $(7,500,000$ control volumes) is shown in Fig. 9(b). The shocks have been implicitly targeted and refined to propagate the signal to the pressure integral surface. The anisotropy of the grid, based on the Mach Hessian, is clearly evident. This anisotropy reduces the number of required control volumes.

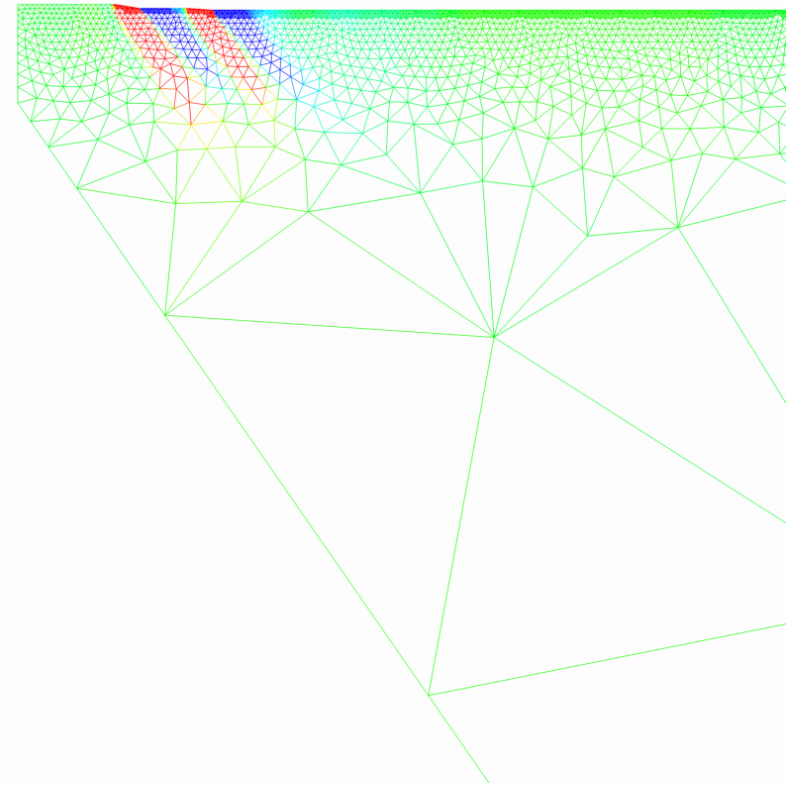

(a) Initial grid.

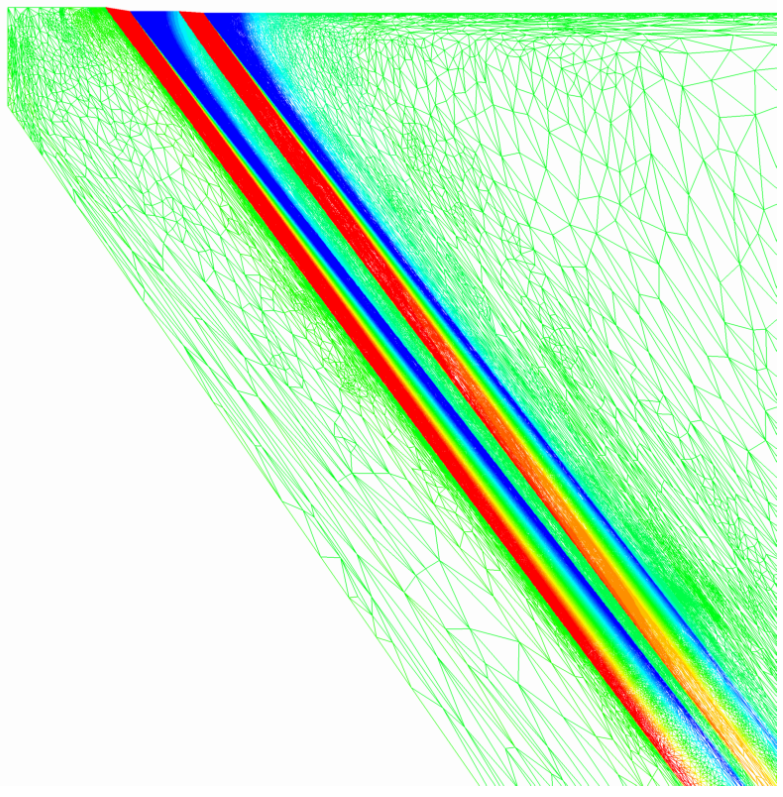

(b) Output adapted grid.

Figure 9. Symmetry planes of initial and output adapted double cone 3D volume grids.

The adaptation history of the pressure integral and its remaining error estimation is shown in Fig. 10. The remaining error estimate is given by Eq. (17). Error is underestimated on the initial few adapted grids before the shocks are propagated to the integration surface. Once this connection is established, the error is reduced.

The adaptation history of the pressure signature extracted at 6 body lengths is shown in Fig. 11. The circular symbols are digitized from a wind tunnel report. ${ }^{52}$ The solid line is the final, adapted signature. The signal is absent on the original coarse grid. The extrema of the pressure signal start to form and grow. The inflection points at $x / l=2.3$ are the last part of the signal to form. The over- and under-shoots of the signal intensify on the final few adapted grids as the grid-shock alignment improves.

The final adapted grid is simulated with the Venkatakrishnan, heuristic, and Barth-Jespersen limiters in Fig. 12. The Venkatakrishnan limiter has similar over- and under-shoots to the heuristic limiter. The Barth-Jespersen limiter produces a signature without over- and under-shoots. All of these limiters have very similar signatures except at the discontinuities.

\section{VI.B. Delta-Wing Configuration}

A delta wing body, denoted "Model 4" in a 1973 wind tunnel report, ${ }^{53}$ is shown in Fig. 13 with the triangular surface grid. This case has also been used by other researchers to evaluate their techniques. ${ }^{54-58}$ The delta wing body was simulated at Mach 1.68 and 0.0 deg angle of attack. The cylindrical integration surface has a radius of 3.6 body lengths and is clipped fore $(x / l=4.3)$ and aft $(x / l=6.6)$ to restrict its extent to the location of the wind tunnel data. These clipping locations are 0.6 body lengths ahead of and 1.7 body lengths behind of the free stream Mach cone emanating from the nose. The width of the integration 


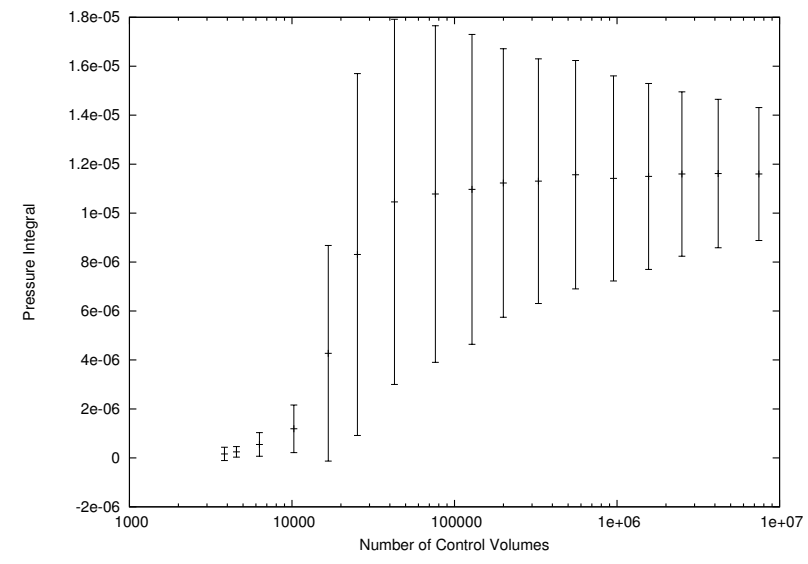

(a) Pressure integral.

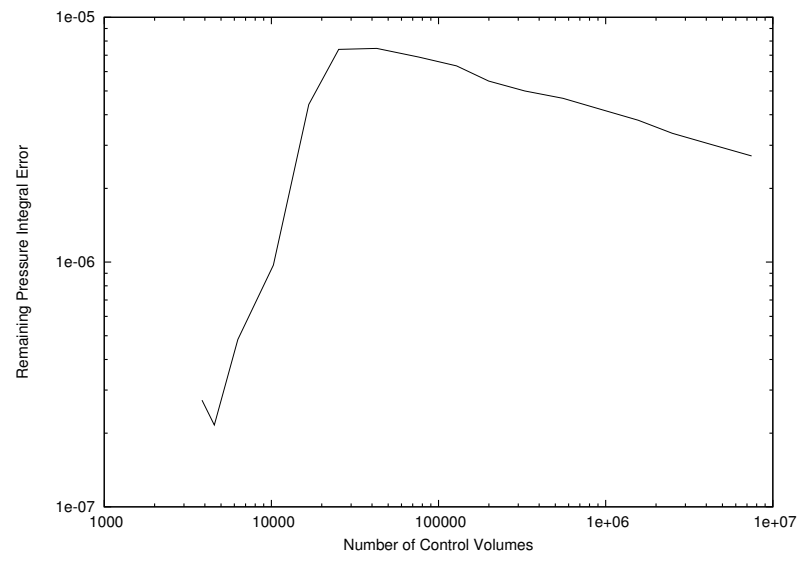

(b) Pressure integral remaining error.

Figure 10. Model 8 pressure integral and uncertainty convergence at 6 body lengths.

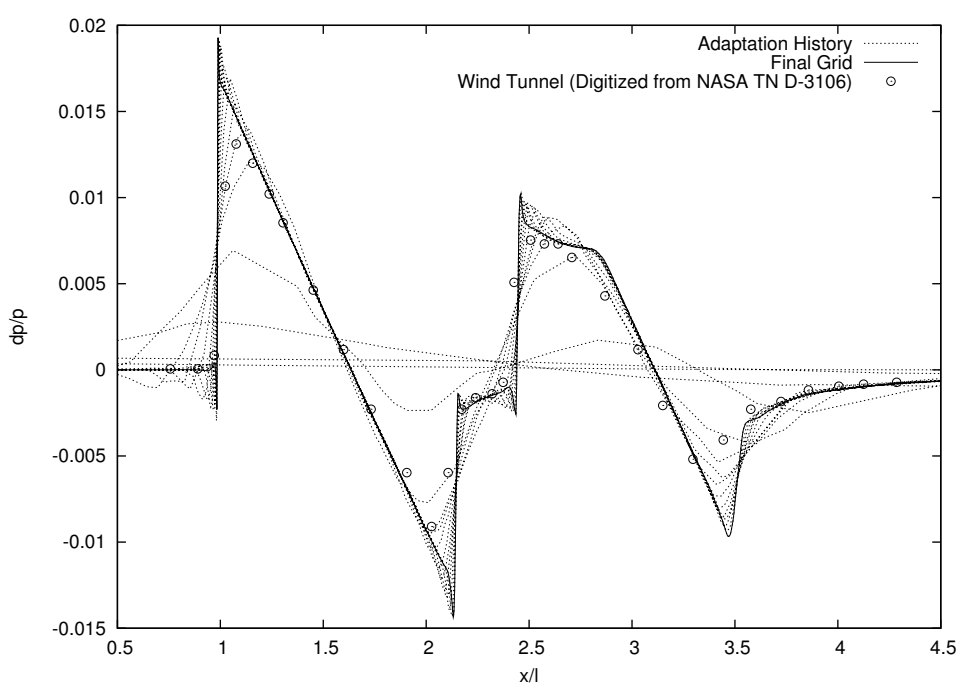

Figure 11. Model 8 pressure signature adaptation history at 6 body lengths.

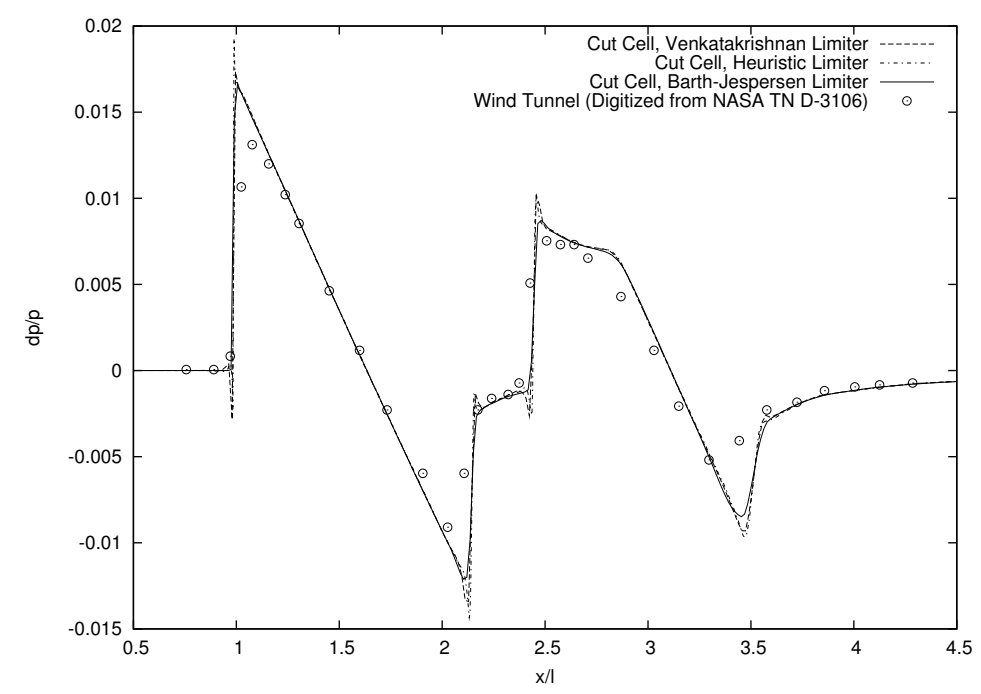

Figure 12. Model 8 final adapted pressure signature at 6 body lengths for various limiters. 


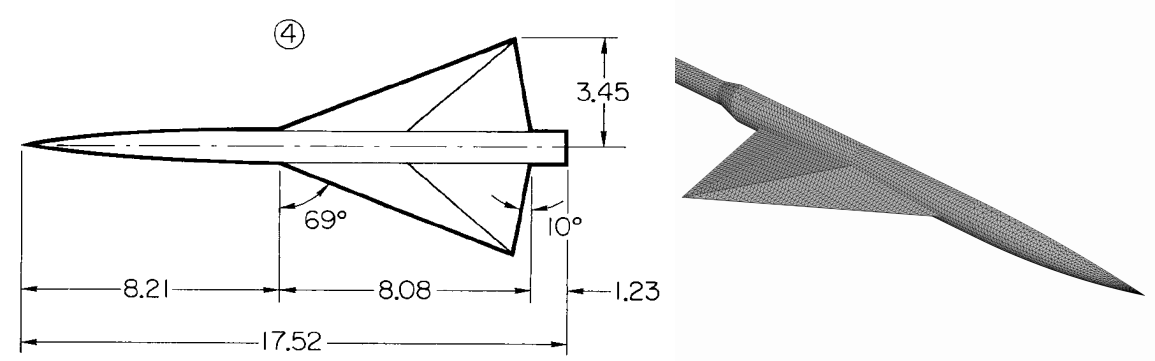

Figure 13. Delta Wing Geometry.

surface is clipped to half a wingspan. Figure 14 shows the initial (2,800 control volumes) and final adapted $(4,900,000$ control volumes) grids colored with pressure. The background grid exit plane and symmetry plane intersected with the model surface grid is shown. The initial grid is extremely coarse. The model is contained in a small number of initial background grid control volumes. The final adapted grid is well aligned with the propagated signal. The refinement region coarsens very rapidly away from the symmetry plane (the integration surface is only a half wingspan wide).

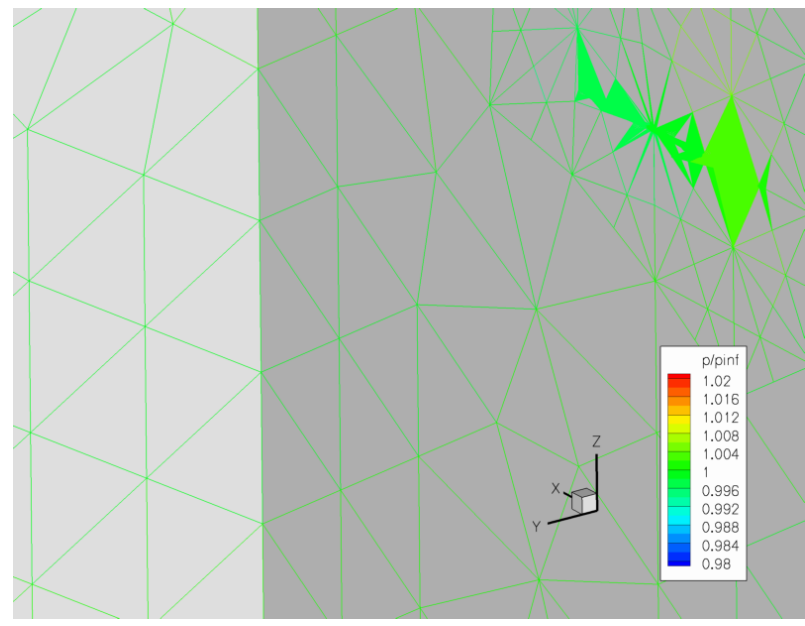

(a) initial grid.

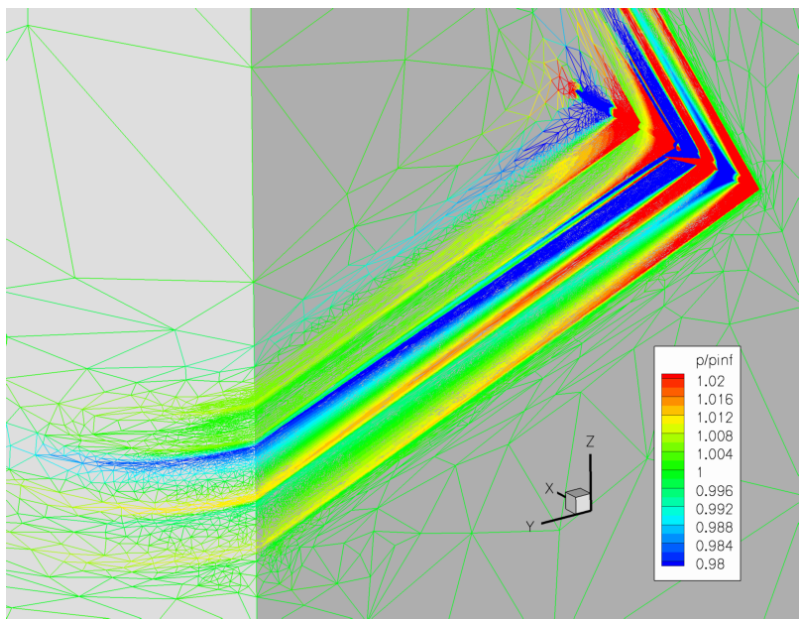

(b) output adapted grid.

Figure 14. Exit and symmetry planes of initial and output adapted delta wing body background grids.

The adaptation history of the pressure integral and remaining error estimation is shown in Fig. 15. Error is initially under predicted for this extremely coarse initial grid. The robustness of the adaptive procedure is illustrated by the use of this coarse initial grid. The remaining error estimate improves as the shocks are resolved and propagated to the integration surface on grids smaller than 100,000 control volumes. This remaining error estimate steadily decreases on grids larger than 100,000 control volumes as the signature is refined. The grid has grown in size by three orders of magnitude.

Figure 16 shows the pressure signatures for the series of grids employed during adaptation. The pressure signature is not apparent on the initial grid. The signals grow as a result of adaptation and the over- and under-shoots increase on the final few grids. The heuristic limiter is employed during adaptation. Figure 17 shows the final adapted grid pressure signatures for the Venkatakrishnan, heuristic, and Barth-Jespersen limiters. The signatures of all three limiters are very similar, except near discontinuities. The BarthJespersen limiter eliminates the over- and under-shoots of the bow and tail shocks. The difference between the Venkatakrishnan and heuristic limiter signatures is very small. 


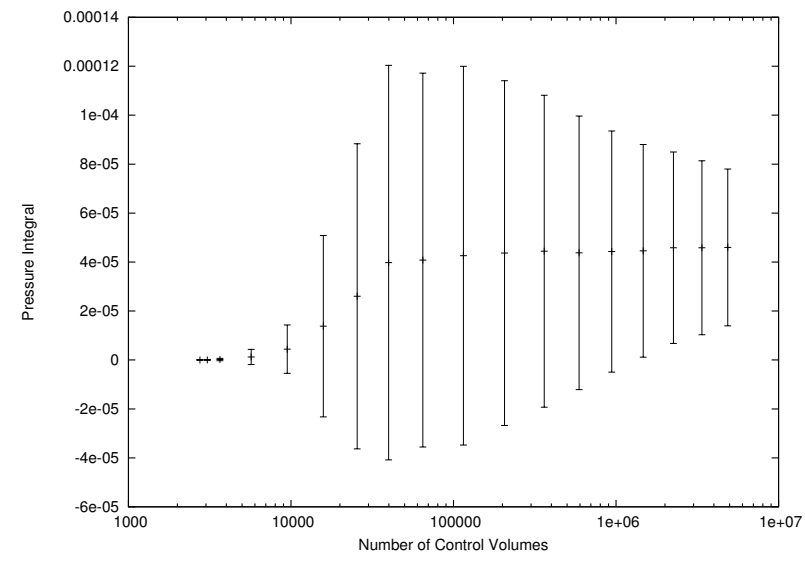

(a) Pressure integral.

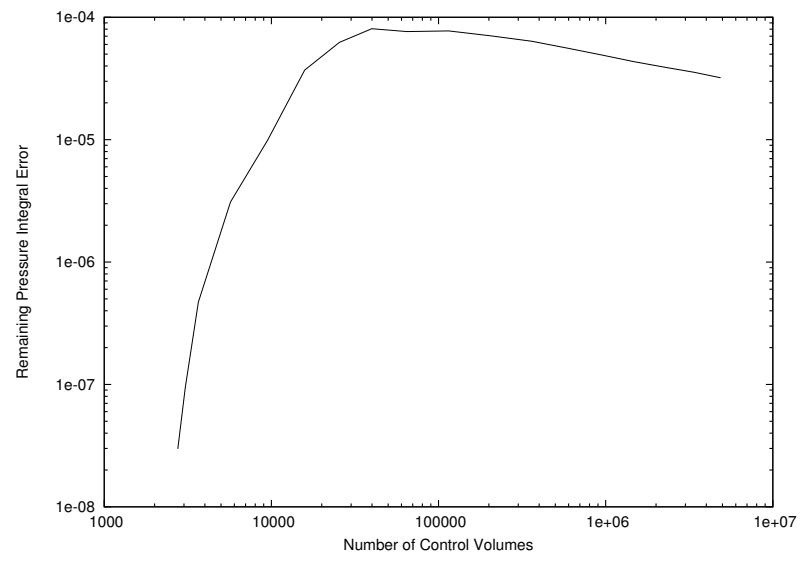

(b) Pressure integral remaining error.

Figure 15. Delta wing body pressure integral and uncertainty convergence at 3.6 body lengths.

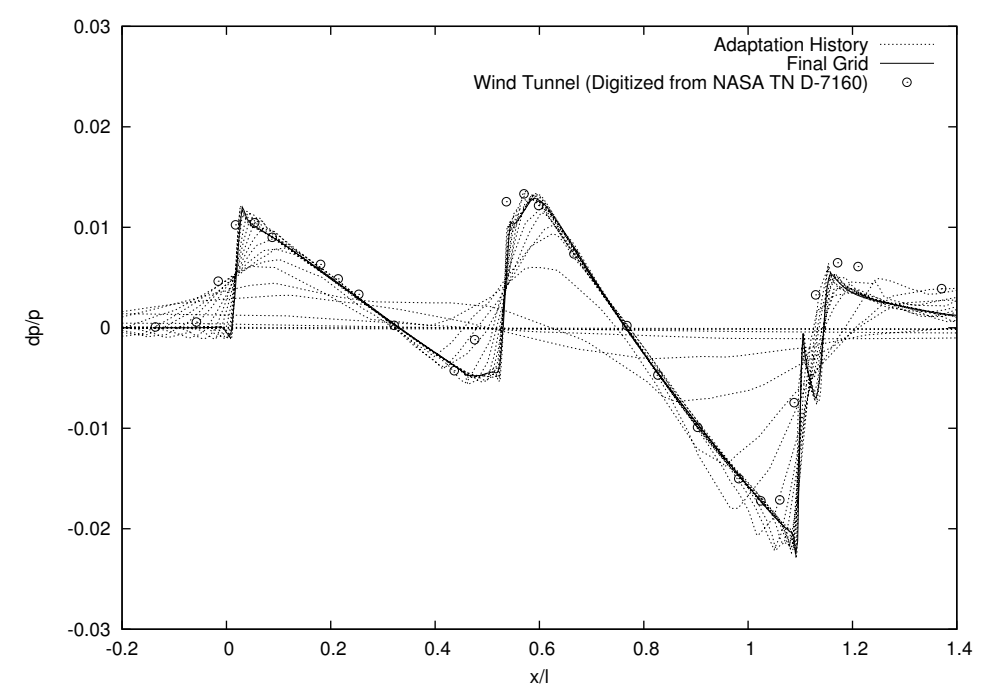

Figure 16. Delta wing body pressure signature adaptation history at 3.6 body lengths.

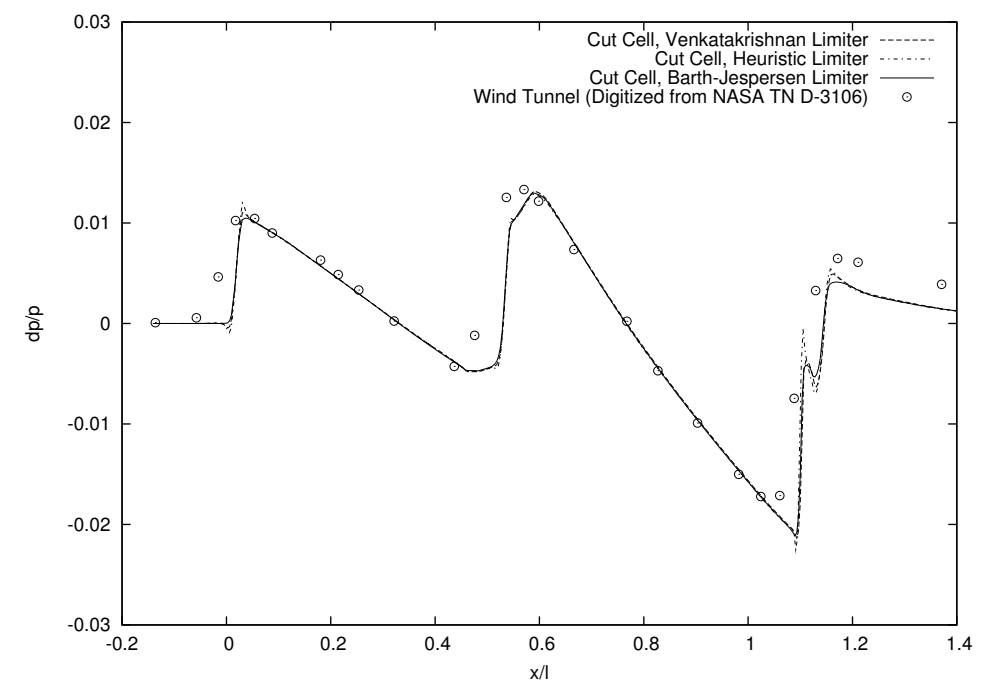

Figure 17. Delta wing body final adapted pressure signature at 3.6 body lengths for various limiters.

\section{2 of 19}

American Institute of Aeronautics and Astronautics 


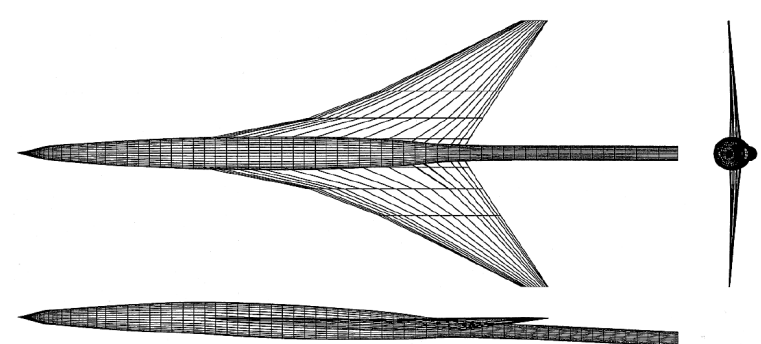

(a) Three-view drawing. ${ }^{59}$

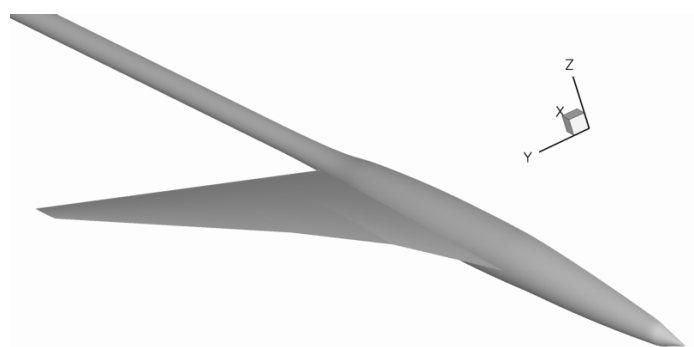

(b) Shaded Surface.

Figure 18. Low-boom wing body geometry.

\section{VI.C. Low-Boom Configuration}

The Straight-Line Segmented Leading Edge (SLSLE) low-boom configuration, Fig. 18, is described by Mack and Kuhn. ${ }^{59,60}$ These reports provide wind tunnel data from two tests, performed at the Langley Research Center Unitary Plan Wind Tunnel Facility ${ }^{59}$ and the John Glenn Research Center $10 \times 10 \mathrm{ft}$ Wind Tunnel Facility. ${ }^{60}$ The test condition is Mach 2.0. The model surface geometry for the aircraft includes the model and sting incidence that provides the wind tunnel lift coefficient $C_{L}=0.08309$ so the angle of attack is zero. ${ }^{11}$ The configuration has a finite thickness trailing edge, which was modeled with a transpiration boundary condition to prevent a strong inviscid supersonic corner flow expansion. Preliminary body-fitted results for this configuration ${ }^{34}$ extended the blunt trailing edge to a sharp trailing edge to avoid the strong supersonic expansion.

The original symmetry plane and cut surface grid colored with pressure is shown in Fig. 19(a). A linear distribution of pressure is shown in each control volume, resulting in a discontinuous pressure distribution on the surface. The initial background grid is isotropic. The final adapted symmetry plane and cut surface grid colored with pressure is shown in Fig. 19(b). The initial background grid contains 40,000 control volumes, and the final adapted background grid contains 5,700,000 control volumes. The anisotropy of the adapted grid has been established with the Mach Hessian, aligning the grid with the shocks.

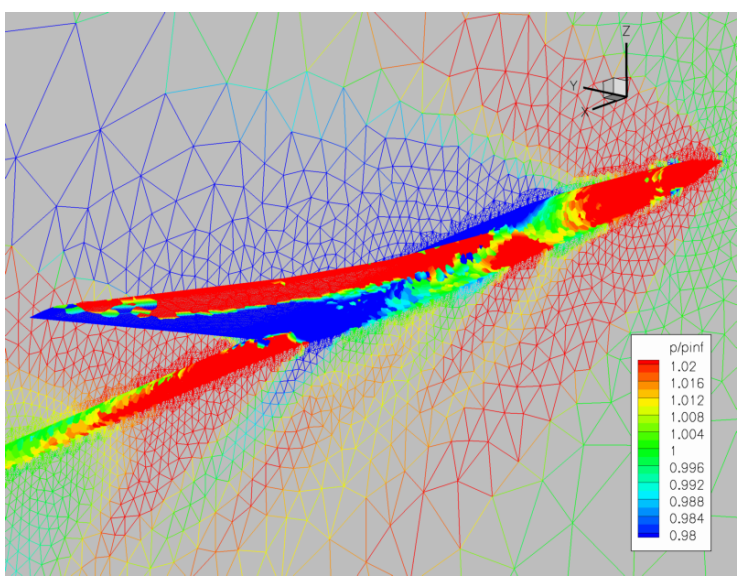

(a) Initial grid.

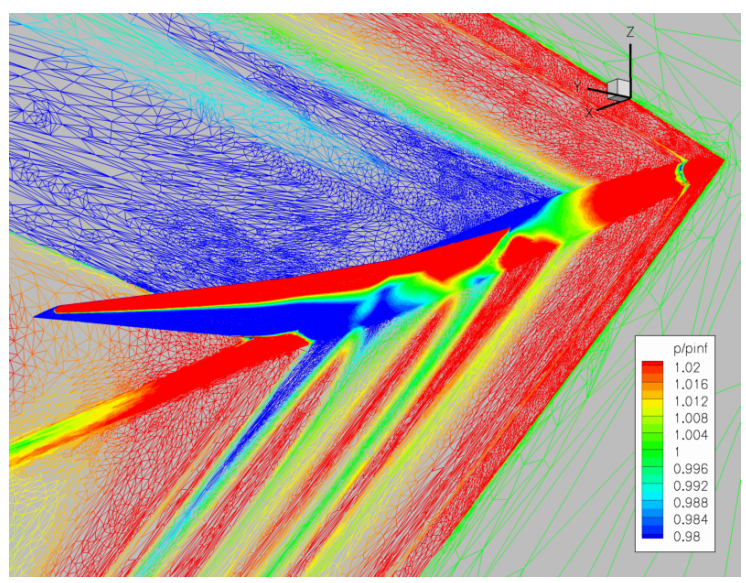

(b) Final grid.

Figure 19. SLSLE surface grid colored with pressure.

The initial, Fig. 20(a), and final, Fig. 20(b), grid integration surfaces are colored with pressure deviation from free stream. These cylindrical integration surfaces are used to compute the output, which drives the adaptation. The cylinder has a radius of 10 body lengths, which is the location of the most distant wind tunnel data that is available. The cylinder is clipped ahead of 32.6 body lengths aft of the model and behind 41.0 body lengths aft of the model. The cylinder is restricted to its lower quadrant. The initial integration surface is poorly resolved due to the initial coarse grid. The pressure signature is not visibly propagated to 
this initial integration surface. The final adapted grid integration surface is a much better approximation of a cylinder due to the background grid refinement. The peak signature pressure is larger at the horizon than the centerline because the model is designed to have a reduced centerline pressure signature.

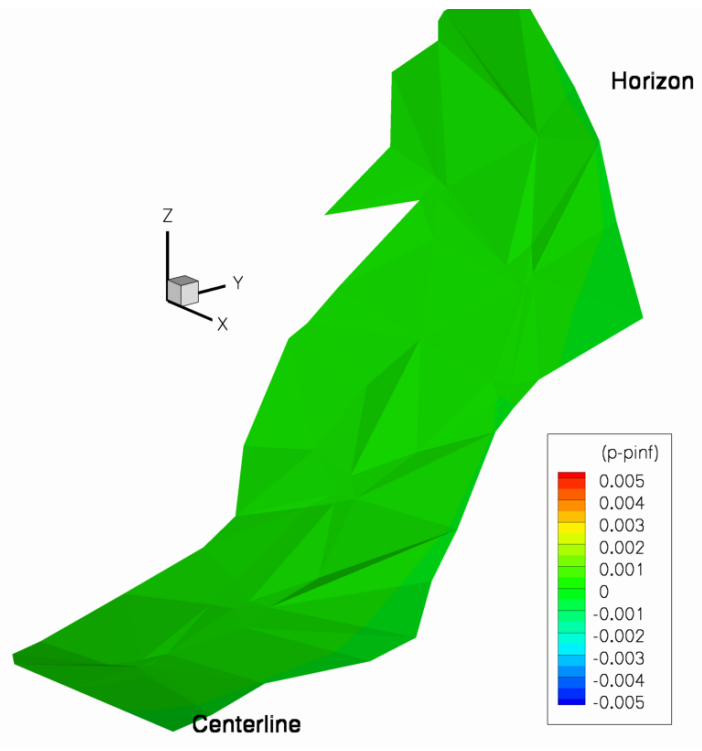

(a) Initial grid.

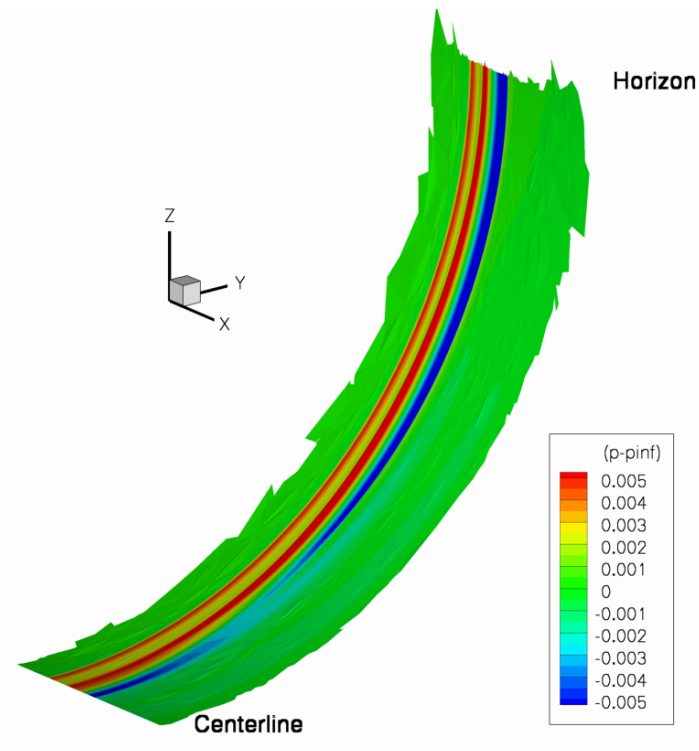

(b) Final grid.

Figure 20. SLSLE pressure on quarter cylinder integration surface 10 body lengths below the model.

The adaptation history of the pressure integral and its error estimation is shown in Fig. 21. The requested error tolerance tol $\Omega_{\Omega}$ is set to half of $I_{\Omega}$ at each adaptive iteration for grids sized less than 2,000,000. Above $2,000,000$ control volumes, the $\operatorname{tol}_{\Omega}$ is set to $I_{\Omega}$, reducing the rate of grid growth. The goal of increasing tol $\Omega_{\Omega}$ is to obtain more resolved results with a more efficiently distributed and aligned grid at the expense of more adaptation cycles. ${ }^{31}$ The change in requested error tolerance is observed as a reduction in grid growth per adaptive iteration in Fig. 21(a). This case shows a less dramatic reduction in the remaining error estimate over the final few grids than the previous cases, which may be due to the less aggressive tol $_{\Omega}=I_{\Omega}$ on the final grids.

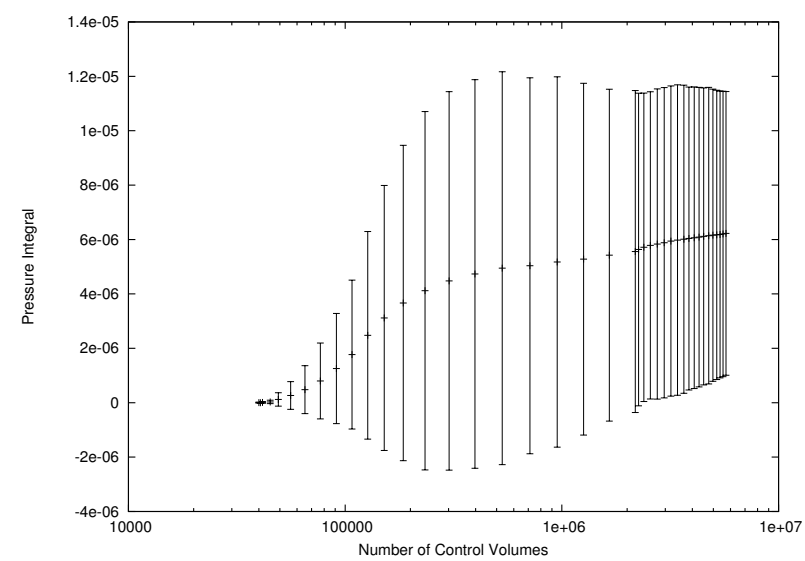

(a) Pressure integral.

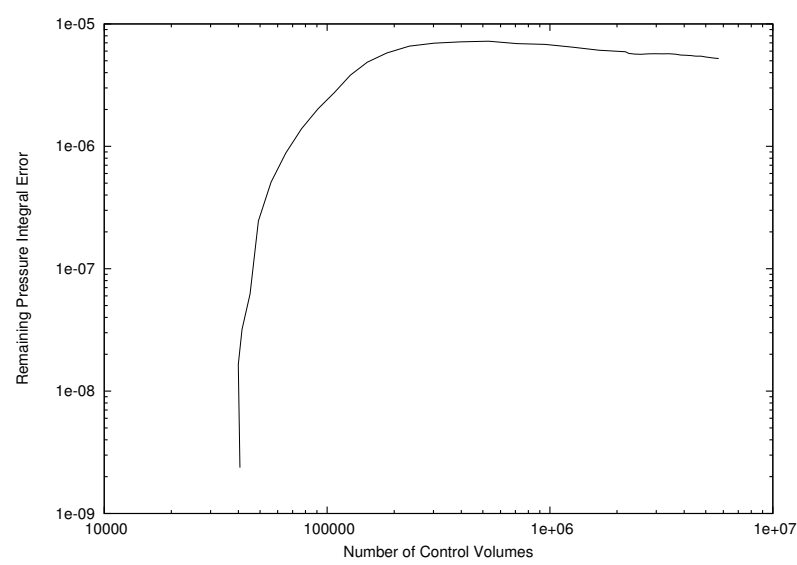

(b) Pressure integral remaining error.

Figure 21. SLSLE pressure integral and uncertainty convergence at 10 body lengths.

The pressure signatures at one body length for the series of grids employed during adaptation is shown in Fig. 22. One body length is much closer than the integration surface, but the signal must be resolved at this location for it to propagate to the integration surface. The pressure signature is broadly smeared on the 
initial grid. The signals grow in amplitude with adaptation and the pressure peaks sharpen on the final few grids. The heuristic limiter is employed during adaptation. The signature mismatch near $x / l=0.8$ is also present at other propagation distances so will be discussed with Fig. 24.

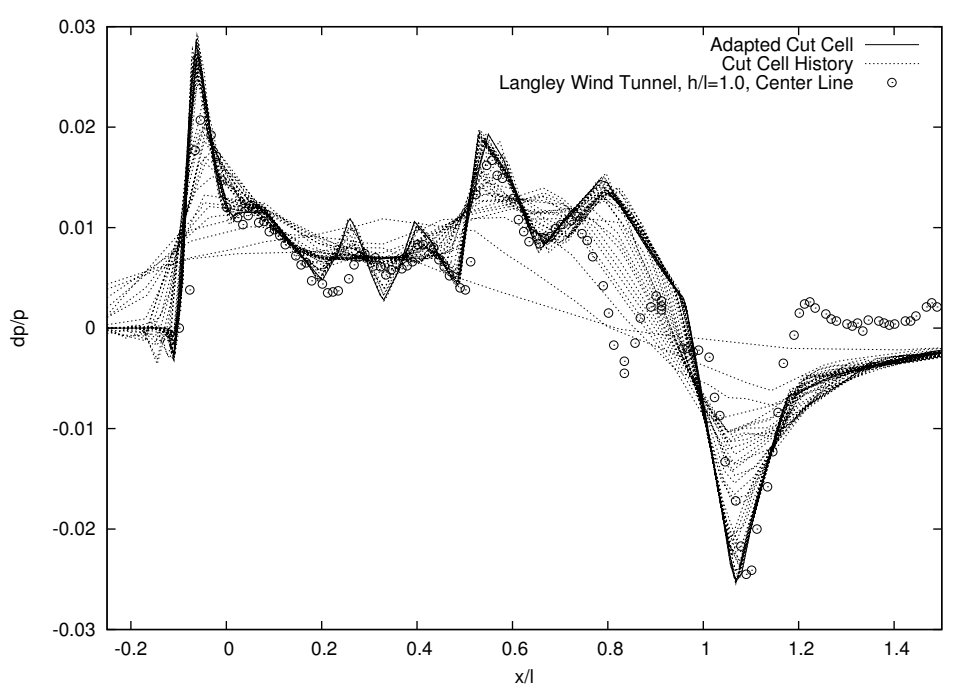

Figure 22. SLSLE pressure signature adaptation history at 1.0 body lengths.

The Venkatakrishnan, heuristic, and Barth-Jespersen limiters are applied on the final grid. The resulting pressure signatures at one body length are shown in Fig. 23. The signatures of all three limiters are very similar, except near discontinuities. The Barth-Jespersen limiter reduces the over- and under-shoots of the bow and tail shocks. The difference between the different limiter signatures is greater for this case than the cone cylinder and delta wing body cases.

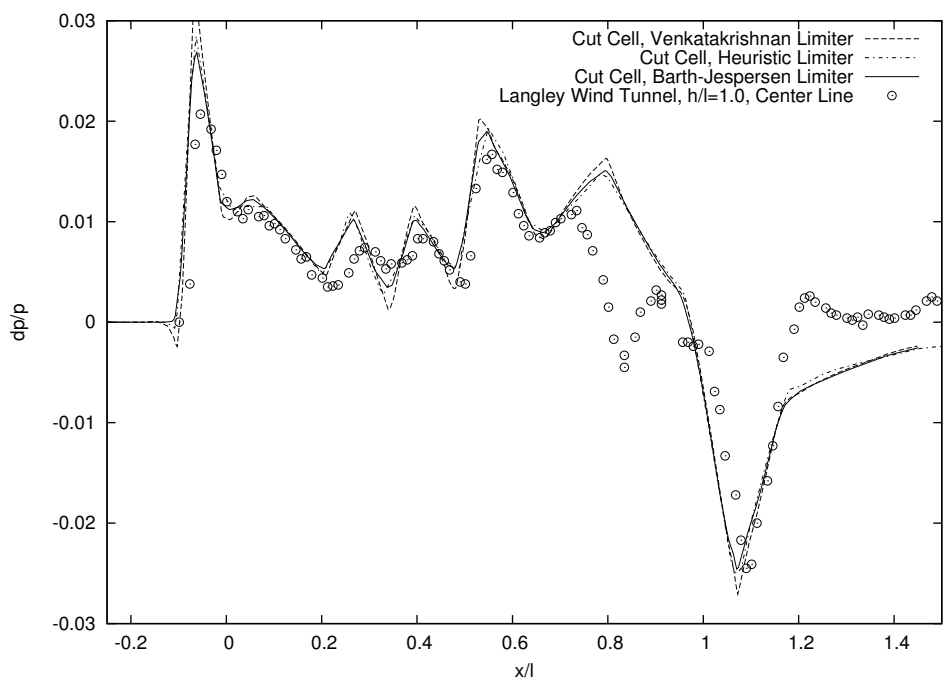

Figure 23. SLSLE final adapted pressure signature at 1.0 body lengths for various limiters.

Centerline pressure signatures are presented in Fig. 24 for 1.0, 1.5, 2.0, and 2.5 body lengths below the model. Langley wind tunnel data is available for all four locations, but the closest Glenn wind tunnel data is available at 2.5 body lengths below the model. The Langley and Glenn wind tunnel measurements are generally in good agreement at 2.5 body lengths in Fig. 24, but the small differences of the two measurements gives an indication of the level of uncertainty in the measurements. The agreement between the wind tunnel and computed signatures is good, except in the region near $x / l=0.8$. Other investigators ${ }^{11,34}$ also showed a difference between wind tunnel and computed pressure signatures at $x / l=0.8$ as well as provided posible explanations. The model geometry in this mismatch region is examined in Park, ${ }^{37}$ which illustrated a 
sensitivity in the signature near an uncertainty in the "as designed" CFD geometry and the "as built" geometry of the wind tunnel model.
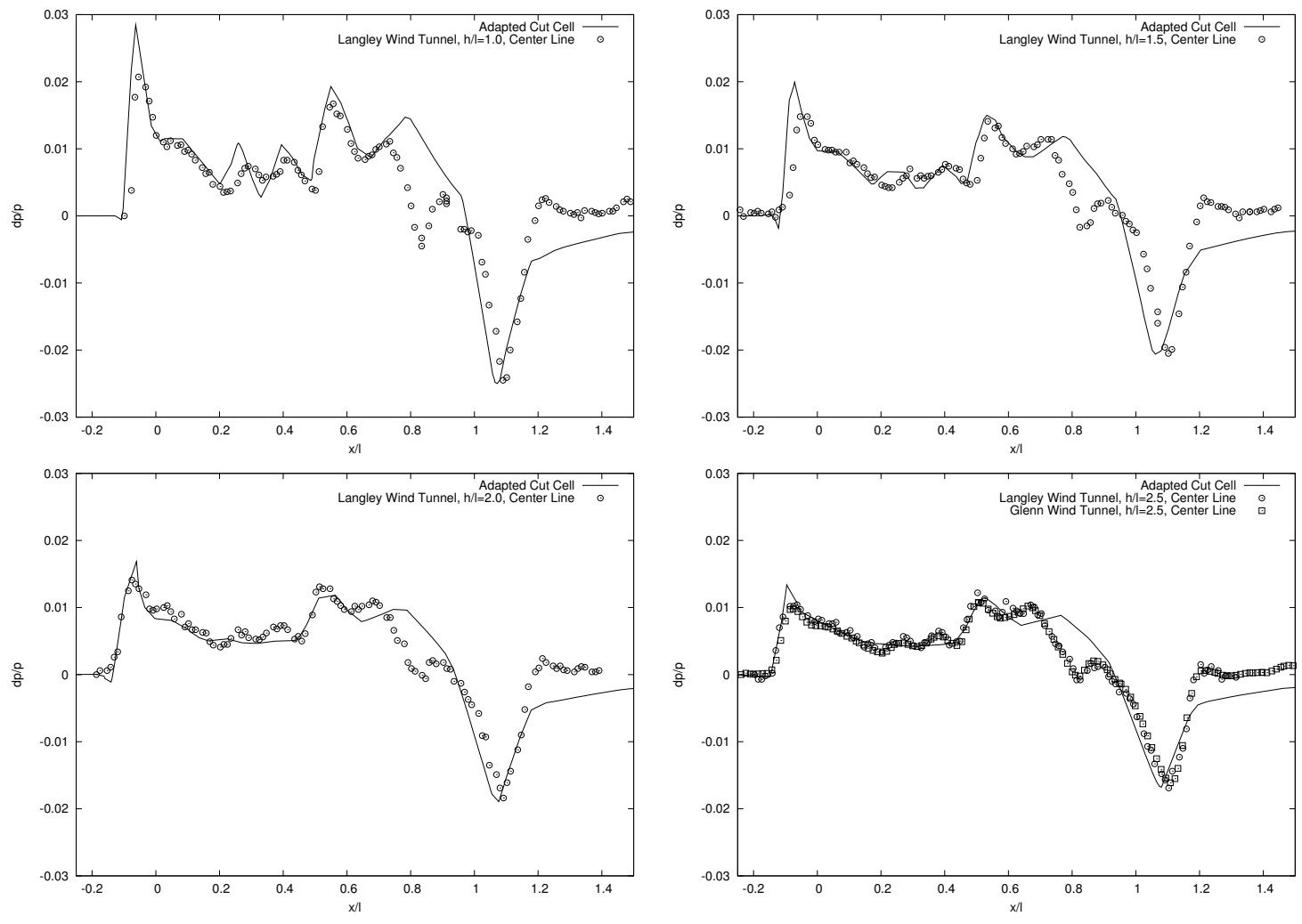

Figure 24. SLSLE centerline pressure signatures for various locations below the model.

Figure 25 compares the adapted cut-cell method with the Glenn wind tunnel measurement at 10 body lengths. This is the same distance as the integration surface. The shock strength increases away from the configuration centerline. The front portion of the signature is well predicted in both Fig. 24 and 25. The aft portion of the computed signal shows the largest difference from the wind tunnel data at all propagation distances. The discrepancy between the wind tunnel and computed signatures near $x / l=0.8$ decreases for the signatures away from the model centerline. The "as built" and "as designed" geometries correspond better laterally.

\section{Summary and Concluding Remarks}

A cut-cell approach to CFD that utilizes the median dual of tetrahedral background grid with a topologically consistent cut-cell geometry calculation method is described. The modifications made to the FUN3D inviscid residual to account for the irregular geometry of the cut cells is detailed. The implications of reconstruction limiting on the discrete adjoint solution are discussed. A single grid output adaptation scheme is described that eliminates the need to generate a memory intensive embedded grid. Output-based adaptation simulations are presented for a cone-cylinder, delta wing, and low-boom configurations to predict off-body pressure signatures in supersonic inviscid flow. These predicted signatures are compared to wind tunnel measurements up to 10 body lengths below the model, providing simultaneous on- and off-centerline predictions. Multiple limiting schemes are applied to illustrate their effects.

The use of cut-cells with an output-based adaptive scheme completely automates this accurate prediction capability after the triangular surface mesh is generated. The use of a heuristic limiter and cut cells with an anisotropically adapted background grid has dramatically improved the robustness of output-based adaptation as applied to sonic boom prediction. This allows extremely coarse isotropic initial grids that can be generated without a priori knowledge of shock locations or Mach angles. The general anisotropy of the adapted background grids allows for centerline and off-centerline signal prediction. This is the first published 

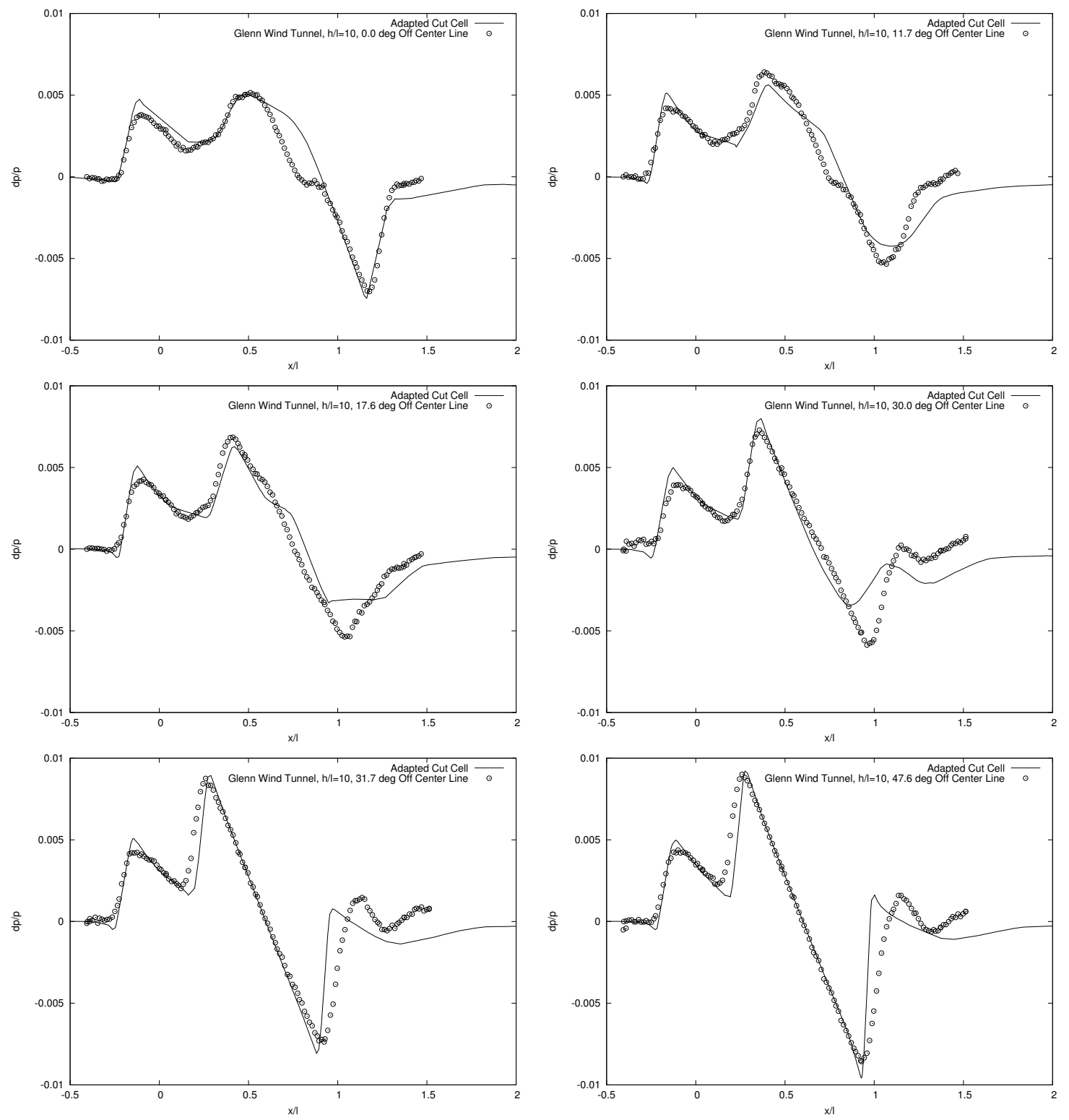

Figure 25. SLSLE pressure signatures for various locations 10 body lengths below the model. 
CFD prediction of the off-centerline SLSLE signatures at 10 body lengths.

\section{Acknowledgments}

A sincere thank you to Jeffery White for his assistance with shock capturing by providing a limiter that enables excellent iterative convergence and to Susan Cliff for providing the surface grid of the delta wing configuration.

\section{References}

${ }^{1}$ Plotkin, K. J., "State of the art of sonic boom modeling," The Journal of the Acoustical Society of America, Vol. 111, No. 1, Jan. 2002, pp. 530-536.

${ }^{2}$ Carlson, H. W., "Experimental and Analytic Research on Sonic Boom Generation at NASA," Sonic Boom Research, edited by A. R. Seebass, NASA, NASA-SP-147, April 1967, pp. 9-23.

${ }^{3}$ Whitham, G. B., "The flow pattern of a supersonic projectile," Communications on Pure and Applied Mathematics, Vol. 5, No. 3, Aug. 1952, pp. 301-348.

${ }^{4}$ Whitham, G. B., "On the propagation of weak shock waves," Journal of Fluid Mechanics, Vol. 1, No. 3, Sept. 1956, pp. $290-318$.

${ }^{5}$ Hayes, W. D., Haefeli, R. C., and Kulsrud, H. E., Sonic boom propagation in a stratified atmosphere, with computer program, NASA-CR-1299, April 1969.

${ }^{6}$ Thomas, C. L., Extrapolation of sonic boom pressure signatures by the waveform parameter method, NASA/TN-D 6832 , June 1972 .

${ }^{7}$ Page, J. A. and Plotkin, K. J., "An Efficient Method for Incorporating Computational Fluid Dynamics Into Sonic Boom Prediction," AIAA Paper 91-3275, 1991.

${ }^{8}$ George, A. R., "Reduction of Sonic Boom by Azimuthal Redistribution of Overpressure," AIAA Paper 68-159, 1968.

${ }^{9}$ Rallabhandi, S. K. and Mavris, D. N., "A New Approach for Incorporating Computational Fluid Dynamics into Sonic Boom Prediction," AIAA Paper 2006-3312, 2006.

${ }^{10}$ Campbell, R., Carter, M., Deere, K., and Waithe, K. A., "Efficient Unstructured Grid Adaptation Methods for Sonic Boom Prediction," AIAA Paper 2008-7327, 2008.

${ }^{11}$ Laflin, K. R., Klausmeyer, S. M., and Chaffin, M., "A Hybrid Computational Fluid Dynamics Procedure for Sonic Boom Prediction," AIAA Paper 2006-3168, 2006.

${ }^{12}$ Kandil, O. and Ozcer, I. A., "Sonic Boom Computations for Double-Cone Configuration using CFL3D, FUN3D and Full-Potential Codes," AIAA Paper 2006-414, 2006.

${ }^{13}$ Waithe, K. A., "Application of USM3D for Sonic Boom Prediction by Utilizing a Hybrid Procedure," AIAA Paper 2008-129, 2008.

${ }^{14}$ Loseille, A., Dervieux, A., Frey, P., and Alauzet, F., "Achievement of Global Second Order Mesh Convergence for Discontinuous Flows with Adapted Unstructured Meshes," AIAA Paper 2007-4186, 2007.

${ }^{15}$ Ozcer, I. A. and Kandil, O., "FUN3D / OptiGRID Coupling for Unstructured Grid Adaptation for Sonic Boom Problems," AIAA Paper 2006-61, 2006.

${ }^{16}$ Peraire, J., Peirò, J., and Morgan, K., "Adaptive Remeshing for Three-Dimensional Compressible Flow Computations," Journal of Computational Physics, Vol. 103, No. 2, 1992, pp. 269-285.

${ }^{17}$ Baker, T. J., "Mesh Adaptation Strategies for Problems in Fluid Dynamics," Finite Elements in Analysis and Design, Vol. 25, No. 3-4, 1997, pp. 243-273.

${ }^{18}$ Aftosmis, M. J. and Berger, M. J., "Multilevel Error Estimation and Adaptive h-Refinement for Cartesian Meshes with Embedded Boundaries," AIAA Paper 2002-0863, 2002.

${ }^{19}$ Rannacher, R., "Adaptive Galerkin Finite Element Methods for Partial Differential Equations," Journal of Computational and Applied Mathematics, Vol. 128, 2001, pp. 205-233.

${ }^{20}$ Pierce, N. A. and Giles, M. B., "Adjoint Recovery of Superconvergent Functionals from PDE Approximations," SIAM Review, Vol. 42, No. 2, 2000, pp. 247-264.

${ }^{21}$ Venditti, D. A., Grid Adaptation for Functional Outputs of Compressible Flow Simulations, Ph.D. thesis, Massachusetts Institute of Technology, 2002.

${ }^{22}$ Nemec, M., Aftosmis, M. J., and Wintzer, M., "Adjoint-Based Adaptive Mesh Refinement for Complex Geometries," AIAA Paper 2008-725, 2008.

${ }^{23}$ Fidkowski, K. J. and Darmofal, D. L., "Output-based adaptive meshing using triangular cut cells," Technical Report ACDL TR-06-2, Aerospace Computational Design Laboratory, Department of Aeronautics and Astronautics, Massachusetts Institute of Technology, 2006.

${ }^{24}$ Barter, G. E., Shock Capturing with PDE-Based Artificial Viscosity for an Adaptive, Higher-Order Discontinuous Galerkin Finite Element Method, Ph.D. thesis, Massachusetts Institute of Technology, 2008.

${ }^{25}$ Nielsen, E. J. and Anderson, W. K., "Recent Improvements in Aerodynamic Design Optimization on Unstructured Meshes," AIAA Journal, Vol. 40, No. 6, 2002, pp. 1155-1163, See also AIAA Paper 2001-596.

${ }^{26}$ Young, D. P., Melvin, R. G., Bieterman, M. B., Johnson, F. T., Samant, S. S., and Bussoletti, J. E., "A locally refined rectangular grid finite element method: Application to computational fluid dynamics and computational physics," Journal of Computational Physics, Vol. 92, No. 1, 1991, pp. 1-66. 
${ }^{27}$ Charlton, E. F. and Powell, K. G., "An octree solution to conservation laws over arbitrary regions (OSCAR)," AIAA Paper 97-198, 1997.

${ }^{28}$ Aftosmis, M. J., Berger, M. J., and Melton, J. E., "Robust and Effcient Cartesian Mesh Generation for Component-Based Geometry," AIAA Journal, Vol. 36, No. 6, 1998, pp. 952-960.

${ }^{29}$ Domel, N. D. and Karman, Jr., S. L., "Splitflow: Progress in 3D CFD with Cartesian Omni-tree Grids for Complex Geometries," AIAA Paper 2000-1006, 2000.

${ }^{30}$ Löhner, R., Baum, J. D., Mestreau, E., Sharov, D., Charman, C., and Pelessone, D., "Adaptive embedded unstructured grid methods," International Journal for Numerical Methods in Engineering, Vol. 60, 2004, pp. 641-660.

${ }^{31}$ Fidkowski, K. J. and Darmofal, D. L., "A triangular cut-cell adaptive method for high-order discretizations of the compressible Navier-Stokes equations," Journal Computational Physics, Vol. 225, No. 2, Aug. 2007, pp. 1653-1672.

${ }^{32}$ Fidkowski, K. J., A Simplex Cut-Cell Adaptive Method for High-Order Discretizations of the Compressible Navier-Stokes Equations, Ph.D. thesis, Massachusetts Institute of Technology, 2007.

${ }^{33} \mathrm{Li}$, X., Shephard, M. S., and Beall, M. W., "Accounting for curved domains in mesh adaptation," International Journal for Numerical Methods in Engineering, Vol. 58, No. 1, 2000, pp. 247-276.

${ }^{34}$ Lee-Rausch, E. M., Park, M. A., Jones, W. T., Hammond, D. P., and Nielsen, E. J., "Application of a Parallel AdjointBased Error Estimation and Anisotropic Grid Adaptation for Three-Dimensional Aerospace Configurations," AIAA Paper $2005-4842,2005$.

${ }^{35}$ Jones, W. T., Nielsen, E. J., and Park, M. A., "Validation of 3D Adjoint Based Error Estimation and Mesh Adaptation for Sonic Boom Prediction," AIAA Paper 2006-1150, 2006.

${ }^{36}$ Jones, W. T., "GridEx - An Integrated Grid Generation Package for CFD," AIAA Paper 2003-4129, 2003.

${ }^{37}$ Park, M. A., Anisotropic Output-Based Adaptation with Tetrahedral Cut Cells for Compressible Flows, Ph.D. thesis, Massachusetts Institute of Technology, 2008, Expected August 2008.

${ }^{38}$ Anderson, W. K. and Bonhaus, D. L., "An Implicit Upwind Algorithm for Computing Turbulent Flows on Unstructured Grids," Computers and Fluids, Vol. 23, No. 1, 1994, pp. 1-22.

${ }^{39}$ van Leer, B., "Flux-Vector Splitting for the Euler Equations," ICASE Report 82-30, 1982.

${ }^{40}$ Nielsen, E. J., Aerodynamic Design Sensitivities on an Unstructured Mesh Using the Navier-Stokes Equations and a Discrete Adjoint Formulation, Ph.D. thesis, Virginia Polytechnic Institute and State University, 1998.

${ }^{41}$ Giles, M., Duta, M., Müller, J.-D., and Pierce, N., "Algorithm Developments for Discrete Adjoint Methods," AIAA Journal, Vol. 41, No. 2, 2003, pp. 198-205, See also AIAA Paper 2001-2596.

${ }^{42}$ Nielsen, E. J., Lu, J., Park, M. A., and Darmofal, D. L., "An Implicit, Exact Dual Adjoint Solution Method for Turbulent Flows on Unstructured Grids," Computers and Fluids, Vol. 33, No. 9, 2004, pp. 1131-1155, See also AIAA Paper $2003-272$.

${ }^{43}$ Barth, T. J. and Jespersen, D. C., "The design and application of upwind schemes on unstructured meshes," AIAA Paper 89-366, 1989.

${ }^{44}$ Elliott, J. K., Aerodynamic optimization based on the Euler and Navier-Stokes equations using unstructured grids, Ph.D. thesis, Massachusetts Institute of Technology, 1998.

${ }^{45}$ Nielsen, E. J. and Kleb, W. L., "Efficient Construction of Discrete Adjoint Operators on Unstructured Grids Using Complex Variables," AIAA Journal, Vol. 44, No. 4, 2006, pp. 827-836, See also AIAA Paper 2005-324.

${ }^{46}$ Venkatakrishnan, V., "Convergence to Steady State Solutions of the Euler Equations on Unstructured Grids with Limiters," Journal of Computational Physics, Vol. 118, No. 1, 1995, pp. 120-130, See also AIAA Paper 93-880.

${ }^{47}$ Balasubramanian, R. and Newman III, J. C., "Discrete Direct and Discrete Adjoint Sensitivity Analysis for Variable Mach Flows," International Journal for Numerical Methods in Engineering, Vol. 66, No. 2, 2006, pp. 297-318.

${ }^{48}$ White, J. A., private communication, 2007.

${ }^{49}$ Park, M. A. and Darmofal, D., "Parallel Anisotropic Tetrahedral Adaptation," AIAA Paper 2008-917, 2008.

${ }^{50}$ Carlson, H. W. and Morris, O. A., "Wind-Tunnel Sonic-Boom Testing Techniques," AIAA Journal of Aircraft, Vol. 4, No. 3, 1967, pp. 245-249.

${ }^{51}$ Morgenstern, J. M., "Wind Tunnel Testing of a Sonic Boom Minimized Tail-Braced Wing Transport Configuration," AIAA Paper 2004-4536, 2004.

${ }^{52}$ Carlson, H., Mack, R., and Morris, O., A Wind-Tunnel Investigation of the Effect of Body Shape on Sonic-Boom Pressure Distributions, NASA/TN-1965-3106, 1965.

${ }^{53}$ Hunton, L. W., Hicks, R. M., and Mendoza, J. P., Some effects of wing planform on sonic boom, NASA/TN-D 7160, Jan. 1973.

${ }^{54}$ Cheung, S. H., Edwards, T. A., and Lawrence, S. L., "Application of Computational Fluid Dynamics to Sonic Boom Near- and Mid-Field Prediction," AIAA Journal of Aircraft, Vol. 29, No. 5, September-October 1992, pp. 920-926.

${ }^{55}$ Djomehri, M. J. and Erickson, L. L., "An Assessment of the Adaptive Unstructured Tetrahedral Grid, Euler Flow Solver Code FELISA," TP 3526, NASA Ames Research Center, Dec. 1994.

${ }^{56}$ Cliff, S. E. and Thomas, S. D., "Euler/Experiment Correlations of Sonic Boom Pressure Signatures," AIAA Journal of Aircraft, Vol. 30, No. 5, Sep.-Oct. 1993, pp. 669-675, See also AIAA Paper 91-3276.

${ }^{57}$ Madson, M. D., "Sonic Boom Predictions Using a Solution-Adaptive Full-Potential Code," AIAA Journal of Aircraft, Vol. 31, No. 1, Jan.-Feb. 1994, pp. 57-63, See also AIAA Paper 91-3278.

${ }^{58}$ Kandil, O., Ozcer, I., Zheng, X., and Bobbitt, P., "Comparison of Full-Potential Propagation-Code Computations with the F-5E "Shaped Sonic Boom Experiment" Program," AIAA Paper 2005-13, 2005.

${ }^{59}$ Mack, R. J. and Kuhn, N., Determination of Extrapolation Distance With Measured Pressure Signatures From Two Low-Boom Models, NASA/TM-2004-213264, 2004.

${ }^{60}$ Mack, R. J. and Kuhn, N., Determination of Extrapolation Distance With Pressure Signatures Measured at Two to Twenty Span Lengths From Two Low-Boom Models, NASA/TM-2006-214524, 2006. 